DOI: $10.2478 / \mathrm{v} 10122-012-0009-\mathrm{x}$

\title{
LEXICA AFROASIATICA VI
}

\author{
GÁBOR TAKÁCS
}

Abstract: Gábor Takács. Lexica Afroasiatica VI. Lingua Posnaniensis, vol. LIV (1)/2012. The Poznań Society for the Advancement of the Arts and Sciences. PL ISSN 0079-4740, ISBN 978-83-7654-103-7, pp. 99-132.

Comparative-historical Afro-Asiatic linguistics has undergone a significant development over the past half century, since the appearence Essai comparatif sur le vocabulaire et la phonétique du chamitosémitique (1947) by Marcel Cohen. This revolutionary and fundamental synthesis concluded the second great period of the comparative research on Afro-Asiatic lexicon (the so-called "old school", cf. EDE I 2-4). During the third period (second half of the 20th century), whose beginning was hallmarked by the names of J.H. Greenberg and I.M. Diakonoff, an enormous quantity of new lexical material (both descriptive and comparative) has been published, including a few most recent attempts (either unfinished or rather problematic) at compiling an Afro-Asiatic compartive dictionary (SISAJa I-III, HCVA I-V, HSED, EHRET 1995).

During my current work on the Etymological Dictionary of Egyptian (Leiden, since 1999-, E.J. Brill), I have collected a great number of new AA parallels, which - to the best of my knowledge - have not yet been proposed in the literature (I did my best to note it wherever I noticed an overlapping with the existing Afro-Asiatic dictionaries). Along the EDE project (and the underlying "Egyptian etymological word catalogue"), I have started collecting AA roots (not attested in Egyptian) for a separate AfroAsiatic root catalogue in late $1999 .{ }^{1}$

The series Lexica Afroasiatica started in $2002^{2}$ in order to contribute to the existing and published materials of comparative Afro-Asiatic lexicon with new lexical correspondences observed recently during my work, which may later serve as basis of a new synthesis of the Afro-Asiatic comparative lexicon. The present part of this series is a collection of new Afro-Asiatic etymologies with the Proto-AfroAsiatic initial bilabial nasal $(* m-)$, which results directly from my research at Institut für Afrikanische Sprachwisenschaften of Frankfurt a/M (in 1999-2000 and 2002) guided by Prof. H. Jungraithmayr. ${ }^{3}$

1 The lexical comparisons presented in this paper issue from a long-range project of an Afro-Asiatic comparative dictionary, and, henceforth, represent the copyright of the author.

2 The first part of this series (lexical parallels with with PAA *b-) has appeared in Afrikanistische Arbeitspapiere (Köln) 67 (2002), 103-151. The second part (additional lexical roots with AA *b-) was published Kogan L. (ed.): Orientalia: Papers of the Oriental Institute, Issue III: Studia Semitica (Moscow 2003, Russian State University for the Humanities, pp. 331-348). My paper Lexica Afroasiatica III (new AA roots with *p-) appeared in Takács G.: Egyptian and Semito-Hamitic (Afro-Asiatic) Studies in Memoriam Werner Vycichl (Leiden 2003, E.J. Brill, pp. 510-550), while Lexica Afroasiatica IV (with new etymologies with AA *f-) was published in Acta Orientalia Academiae Scientiarum Hungariae (Budapest) 57(4) (2004), 457-473. The following part, Lexica Afroasiatica $V$ (new AA roots with $* \dot{p}-, *^{*} P$-) appeared in Studia Etymologica Cracoviensia 9 (2004), 159-178.

3 The author expresses his deep gratitude to Prof. Herrmann Jungraithmayr and the Alexander von HumboldtStiftung (Bonn) for the support of his research at the Institut für Afrikanische Sprachwissenschaften (Frankfurt a/M, Germany) in 1999-2000, 2002, when materials for the present article have been gathered. Thanks are due also to the OTKA (Hungarian National Scientific Research Fund) for supporting my work on the 3rd volume of EDE since 2003. 
The numeration of the etymological entries is continuous beginning from the first part of the series Lexica Afroasiatica.

Each entry is headed by the proposed PAA root (as tentatively reconstructed by myself). Author names are placed after the quoted linguistic forms in square brackets [] mostly in an abbreviated form (a key can be found at the end of the paper). The lexical data in the individual lexicon entries have been arranged in the order of the current classification of the Afro-Asiatic daughter languages (originating from J.H. Greendererg 1955, 1963 and I.M. Diakonoff 1965) in 5 (or 6) equivalent branches: (1) Semitic, (2) Egyptian, (3) Berber, (4) Cushitic, (5) Omotic (cometimes conceived as West Cushitic), (6) Chadic. For a detailed list of all daughter languages cf. EDE I 9-34. The number of vertical strokes indicate the closeness of the language units from which data are quoted: || separate branches (the 6 largest units within the family), || groups (such as East vs. South Cushitic or West vs. East Chadic), while | divides data from diverse sub-groups (e.g., Angas-Sura vs. North Bauchi within West Chadic).

Since we know little about the Proto-Afro-Asiatic vowel system, the proposed list of the reconstructed Proto-Afro-Asiatic forms is arranged according to consonantal roots (even the nominal roots). Sometimes, nevertheless, it was possible to establish the root vowel, which is given in the paper additionally in brackets. The lexical parallels suggested herein, are based on the preliminary results in reconstructing the consonant correspondences achieved by the Russian team of I.M. Diakonoff (available in a number of publications ${ }^{4}$ ) as well as on my own observations refining the Russian results (most importantly TAKÁCS 2001). The most important results can be summarized as follows. The labial triad ${ }^{*} b-{ }^{*} p-{ }^{*} f$ remained unchanged in Egyptian, South Cushitic, and Chadic, while the dental series $* d-* t-{ }^{*} t$ was kept as such by Semitic and South Cushitic (AA *t continued as * $d$ in Berber, Cushitic and Chadic, and it was merged into $t$ vs. $d$ in Egyptian). The fine distinction of the diverse sibilant affricates and

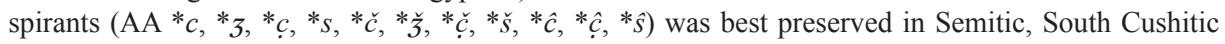
and West Chadic (while some of these phonemes suffered a merger in other branches and groups). The Russian scholars assumed a triad of postvelar (uvulear) stops with a voiceless spirant counterpart: ${ }^{*} g$, $* \dot{q},{ }^{*} q$, and $* h$, the distinction of which was retained in Cushitic and Chadic, but was merged into $* h$ in Semitic and Egyptian. In a number of cases, however, it is still difficult to exactly reconstruct the root consonants on the basis of the available cognates (esp. when these are from the modern branches, e.g., Berber, Cushitic-Omotic, or Chadic). In such cases, the corresponding capitals are used (denoting only the place of articulation) ${ }^{5}$

Gábor Takács, Institute of Linguistics, Hungarian Academy of Sciences, Dept. of Egyptology, ELTE, Székesfehérvár, Hungary

475. AA * $\boldsymbol{m}$ "in, from" > Eg. $m$ "1. in, 3. from, out of" (OK, Wb II 1-2; FD 99; EG 1927, $\S 162) \rightarrow$ adv. jm "da, dort, dorthin" (OK, Wb I 72) = "there(in), therewith, therefrom" (FD 17) ||| Sem.: perhaps Geez ?am "from, out of (place) etc." [Lsl. 1987, 22] and Gafat ammä $\sim m \ddot{a}$ “à, dans (lieu, temps), de" [Lsl. 1956, 176] ||| Agaw: Kemant - $\eta \bar{a}$ [reg. <*mā] (postp.) "dans" [CR 1912, 239] ||| NOm.: Benesho $-m \sim-n$ "on, at, in" [Wdk. 1990, 115] ||| WCh.: Fyer ? $m \sim m$ "nach, zu, an, in" [Jng. 1970, 87] | Kupto -m-/-m "in”, cf. mà "von" [LeGER 1992, 20, 27] || CCh.: Glavda mà má “in, from, within, out of” [RB 1968, 61], Mnd. ám (preverb) "hinein, herein" [WolfF 1974, 19], Dghwede me "in, into, apart" [FRICK 1976, 8] || ECh.: Mubi má “1. her von, 2. beim Komparativ, 3. Und” [Lks. 1937, 184]. Here might

The first draft of the present paper was prepared in 2003. A thorough updating and some additions have been made in autumn 2006.

4 Cf. Diakonoff 1984, 1988: 34-41, 1992, Diakonoff et al. 1987, 1993, SISAJa I-III; HCVA I-V.

5 E.g., the symbol $(* P$-) signifies any unknown initial labial, just as, e.g., $* T$ stands for any unknown dental stop $(* d, * t$ or $* t)$ or $* K$ for any unknown velar stop $(* g, * k$ or $* k)$ or $* Q$ for any unknown postvelar/uvulear $(* g, * q$ or $* \dot{q})$, while $* H$ for any laryngeal or pharyngeal $(* \eta, * h, * \rho, * h$, also $* \gamma)$. 
belong the Akk. adv. case ending -um (Djk.: - $m$ here was not the mimation) which I.M. DiAKONOFF $(1988,61)$ took from AA * $V m$ "locatativus-adverbialis".

476. AA * $\boldsymbol{m}$ "with $\rightarrow$ by means of" $>$ Eg. $m$ " 2 . mit" $(\mathrm{OK}$, Wb II 1-2) $=$ " 2 . with, by means of, together with" (FD 99; EG 1927, §162) ||| Om.: cf. perhaps Ongota (if it belongs with Om.) $-m i \sim-m e$ "by (means of), with (noun suffix, agentive or instrumental)" [Flm. 1992, 212] ||| WCh.: Sura mós (prep.) “and, with” [Jng. 1963, 75], isolated in AS (GT 2004, 250)| Kupto má “zusammen, mit" [LEGER 1992, 20] || CCh.: Hide (Htk.) ma “de, à partir de, dans, à” [EGUCHI 1971,216]|Mbara máy" "with”[TSL 1986, 271, 284] || ECh.: Mgm. má “avec"[JA 1992, 105].

477. AA *m (prep.) "as, like" > Eg. $m$ (der Identität) “als" (OK, Wb II 1-2) = “4. as, namely (m of predication)" (FD 99; EG 1927, \#162) is cognate with NBrb.: Ikhebdanen \& Iqrayen \& Temsaman \& Uriaghel am "comme, ceque" [BIARnAY 1917, 83], Sgrs. am "comme" [Pellat 1955, 103], Izn. \& Bqy. \& Amr. am "comme" [Rns. 1932, 382] = am "comme, environ" [ABÈs 1916, 110], Bettiwa am "comme" [Brn. 1911, 183], Mzg. am(m) “(indique la comparaison, la manière) comme, tel (que), à l'instar de, 2. aussi bien que, aussi ... que" [Taïri 1991, 398], Ait Said $m$ "quand, comme" [Allati 1986, 10], Mzab am an (prép.) "comme” [Dlh. 1984, 113], Wrg. am "comme" [Dlh. 1987, 181], Zayan \& Sgugu am “comme" [Lbg. 1924, 563], Nfs. am "come (comparativo di uguaglianza)" [Bgn. 1931, 259] | Qbl. am (prep.) "comme” [Dlt. 1982, 478] || EBrb.: Siwa \& Sokna am "comme” [Lst. 1931, 217], Audjila am "come (avverbio di maniera)" [Prd. 1960, 163] || SBrb.: Hgr. am "as, like" [Prasse 1972, 230] ||| WCh.: Krkr. mú "Partikel am Ende des Temporalsatzes: als" [Lks. 1966, 230] || CCh.: Mbara mày "comme, aussi” [TSL 1986, 271] || ECh.: Mubi má "beim Komparativ" [Lks. 1937, 184]. Cf. also LECu.: Som. may “Gleichnis” [Rn. 1902, 306].

478. AA * $\boldsymbol{m}$ "when, while" [GT] > Eg. $m$ "5. (before suff. conj.) when, as, though etc." (FD 99; EG 1927, \#162) ||| NBrb.: Warsenis \& Tarudant ma "lorsque" \& Shawya ma "si" [Bst. 1890, 310], Bettiwa mi "lorsque" [Brn. 1911, 183], Mzab mmi "quand, lorsque" [Dlh. 1984, 114], Wrg. mmi "quand, lorsque" [Dlh. 1987, 182] | Qbl. mi "lorsque, quand" [Dlt. 1982, 477], Zwawa ma "si" [Bst. 1.c.] etc. || SBrb.: Hgr. emmi [Fcd. 1951-2] || CCh.: Bura ma "if, when" [BED 1953, 126], Margi mà "1. if, when, 2. Before" [Hfm. in RK 1973, 123] | Malgwa má "wenn, falls" [LöHr 2002, 212], Glavda ma "if” [RB 1968, 61] || ECh.: Mgm. máa "lorsque, quand" [JA 1992, 105].

479. AA * $\boldsymbol{m}$ (act. *ma?) (particle of emphasis) > Sem. *-ma (emphatic part.) [GT]: Akk. -ma "hervorhebende Partikel und Konjunktion: 1. zur meist identifizierenden Heraushebung einzelner Wörter", cf. - $m i \sim-m e$ (all) "wird in zitierter direkten Rede an betonte Wörter angehängt" [AHW 560, 650] || Ar. $m \bar{a}$ "Partikel zum Nachdruck", e.g. yā 'ayni fā-bki mā ban̄ asadi "O mein Auge beweine die Bani Asadi” [Nöldeke apud Ember] || ES: Geez -mma "particle of emphasis: precisely, quite, then" [Lsl. 1987, 323], Har. -ma "as to, indeed" [Lsl. 1963, 102] ||| Eg. $m$ (encl. part.) "doch: 1. nach einem Imperativ (z.B. sage doch, komme doch!), 2. jw $m$ (MK) als Satzanfang in Briefen" (OK, Wb II 36, 6-7) = "(nach Imperativ, selten davor) bitte, dich!" (GHWb 323) = "please, pray" (DLE I 210) || WCh.: Goemai $m a$ "indeed" [Srl. 1937, 132] | cf. perhaps Dera àmmá "but" [Nwm. 1974, 121] || CCh.: Gisiga ma "verstärkerndes ma: doch, auch" [Lks. 1970, 127] || ECh.: Mokilko mê, mè "Verstarkungspartikel" [Lks. 1975, 224].

Lit.: The Eg.-Sem. comparison was first proposed by A. EMBER (1914, 303-304, \#2). 
480. AA * $\boldsymbol{m}$ or * $\boldsymbol{m}$-y "person": Brb. * $i$-m-an "soul(s)" (pl. affixes $*_{i-\ldots}$-an attached to the orig. root*m) [GT]: NBrb.: Mzab iman "âme, esprit, soi" [Dlh. 1984, 114], Wargla iman "âme, personne" [Dlh. 1987, 182], Izn. imän "âme, vie, personne" [Rns. 1932, 390], Qbl. iman [Dlt. 1982, 503] || EBrb.: Siwa \& Gdm. \& Nfs. iman "âme" [Lst. 1931, 194] || SBrb.: Hgr. iman [Fcd. 1951-2, 1138], EWlm. \& Ayr iman "1. âme(s), 2. principe de vie, 3. personne" [PAM 1998, 207; 2003, 517] (Brb.: NZ 1998, 140-1, §128) ||| ECu.: Dasenech (Galab) $m u ́$ "homo", $m \bar{a}$ "vir" [SASSE 1974, 422] = máa "person, man", $m i ́ \sim m u ́$ "man, person" [Tosco 2001, 516] || SCu. *mi "people" [Ehret] = *mV, orig. perhaps *mu [GT]: WRift: Irq. \& Grw. mu (pl.) "people (persons)" [Wtl. 1958, 24], Qwd. me?-iko “people” [Ehr. $1980 \mathrm{MS}$, 4] | Ma'a m-mú "person", va-mú "people” [TB 1974, 193; Ehr. 1974 MS, 46; Mous 1996, 207: < Irq.] (SCu.: Ehret 1980, 160, \#55) ||| NOm.: Wolayta moy-tilliya \& Gamu \& Zayse moy-tille "ghost" [Lmb.] | Yemsa (Janjero) $m \bar{e}$ "vielleicht Menschen, Frauen" [Lmb. 1993, 362] | Shinasha mèyá "Geist" [Lmb.] | Sezo may "man (vir)" [Bnd.] = mj̀: "man" [Sbr.Wdk.], Hozo mōó "uomo" [Grt. 1940, 356] = mo "man (vir)" [Bnd. 1990] = "person" [Bnd. 1994, 1159, \#62] = mo: "man" [Sbr.-Wdk.] (Mao: Bnd. 1990, 610, \#148; Sbr.-Wdk. 1994, 14; NOm.: Lmb. 1993, 364) ||| WCh. *m[a]y- "person" [GT]: PGmy. *may (with prefix *kzsg. vs. *mo- pl.) "man" [GT 2004, 243-4]: Gmy. goe-mai "an inhabitant of the Ankwe land" [Srl. 1937, 64] = mó-mai (pl.) "Mann, Mensch" [Jng. 1962 MS, 2] = gə-mai "Goemai”, mo-mai (pl.) "people" [Hlw. $2000 \mathrm{MS}, 11,22$ ] | BT *mu/*mi, pl. *miya "person" [Schuh] $=* m-m$ "man (vir), male, person" [JS 1981, 174: A $/ 2$ ] = *mUy- * miy- (sg.) "person" \& *mī-mi * *miya (pl.) "people" [GT]: Bole méémù (sg.), mi’?yà (pl.) [Schuh] = me:mu "vir, person" [IL] = memu "person" [Meek], Tangale miye (pl.) "Menschen" [Jng. 1957, 149] = тии ти "Mann, Person" [Jng. 1991, 122], Bele móyyò (sg.), mìmò (pl.) [Schuh], Maha mòo "people" [Alıo 1988 MS], Kirfi mímí (sg.), mì̀ní (pl.) "person" [Schuh], Galambu mì̀ (sg.) [Schuh] = míi "person(al spirit)" [Alıo 1988 MS], Geruma mími (sg.), mùnú (pl.) "person" [Schuh], Dera mu "home" [Pls. 1958, 78] = mú, pl. míyá [Schuh] = mami "Mann", múù "Mann, Mensch, Person", miya "Leute, Volk" [Jng. 1966 MS, 10-11] = máámí "husband, male" [Nwm. 1974, 129] = múù "person (homo), man" [KidDA 1991 MS, 1], Kwami múu, pl. míyá "Mensch, Person" [LEGER 1992, 28; 1993, 172] = "human being, person" [Jng.-Leger], Krkr. mán (pl.) [Schuh], Kupto múu, pl. míyá “Mensch, Person” [Leger 1992, 21], Kushi mèmé (pl.) "human being, person" [Jng.-Leger], Piya míyá (sg.) "person" [Jng.Leger] (BT: Schuн 1978, 150; 1984, 211; Jng.-Leger 1993, 167) | Guruntum mu "man, person" [Jgr. 1989, 186-7], Jimi ma "Mann" [Gowers], Bubure múmó "person (homo)", mìmmìýc "people" [HARUnA 1992 MS, \#a001-2], Dokshi mii "person” [Smz. 1978, 29, \#39] (WCh.: JI 1994 II, 230) || CCh.: Bata meye "personne" [Mch.] = m(y)á "person, s'one" [Pweddon 2000, 52] = ma "(male) person" [Boyd 2002, 56] | Logone mee $\sim$ meea (in gen. cstr.: mi mii miia miie) "Leute" [Nct. apud Lks. 1936, 108-9], Buduma mwey "home" [Gaudiche 1938, 20], Gulfei meiwwe (pl.) "Männer" [Lks. 1937, 150], Ngala moi "people" [Mgd. 1922, 236] (W-CCh.: JI 1994 II, 266-7) || ECh.: Gabri k̇̀-mā "personne” [Cpr. 1972 MS]. For the AA etymology see also Mkr. 1981, 210, \#38.A (SCu.-Mao); 1987, 253 (BTMao); Orel 1995, 108, \#119 (PWCh.-POm.-PRift).

NB1: A.B. Dolgopolsky $(2005,26)$ reconstructs PBrb. *-mān "soul, person" Eg. $m n$ "a such-and-such"< Nst. *me[y] ñU "o'self, one's own, body". Improbable.

NB2: JI (1994 I, 115) explained the Ch. forms from PCh. *m-t-m, while R.M. VoIGT $(1998,612)$ erroneously assumed a derivation of the them either (!) from PCh. * $m-t$ or * $m-n$ "Mann". 
481. AA * $\boldsymbol{m}$ - $\boldsymbol{d}$ "to press": SBrb.: Hgr. $e-m d i$ "enfoncer sous terre près de la surface du sol" [Fcd. 1951-2, 1160] ||| LECu. *m-d "to (op)press" [GT]: Oromo mudda "to press, tighten, torment" [GRAGG 1982, 292], Oromo-Borana mudda mudd-ita "to press, tighten, frighten, scare, disturb" [Strm. 1995, 211] | Rendille a-mída "ich belästige, bedränge, dominiere mit Gewalt" [SchleE 1978, 140, \#773] | HECu.: Sidamo mudda "to hasten, hurry (tr.), press" [Gsp. 1983, 239]. A var. root to AA *m-t "to press" (below).

482. AA * $\boldsymbol{m}$ - $\boldsymbol{d}$ "to work, serve": LECu.: Som. midìdin "servant, assistant" [Abr. 1964, 179] ||| NOm.: Omt. mad- "lavorare, servire, ministrare, aintare" [Mrn. 1938, 143] || Ch. *m-d "slave" [JS 1981, 236: B4] > CCh.: Gisiga modro "Diener" [Lks. 1970, 130], Balda(mu) mắdai "Sklave" [Str. 1922-23, 119; not recorded by Trn. 1987, 54] || ECh.: Tumak mààd "esclave" [Cpr. 1975, 81].

NB: Not clear if NEg. $m 3 d j . w$ (provided it was a group-writing for * $m d j . w$ ) (pl.) "Diener, die Speisen zutragen" (XIX., Wb II 35, 5) = "waiters, servers (of food)" (DLE I 209) = "Diener, Aufwärter" (GHWb 322) also belongs here.

483. AA * $\boldsymbol{m}$ - $\boldsymbol{d}$ "to help, protect" > Ar. madda I "10. prêter assistance à qqn., l'aider avec qqch.", IV "2. aider, assister qqn., lui donner des secours (en troupes, en vivres, etc.); envoyer des troupes auxilières à qqn.", X "1. demander des secours, de l'assistance chez qqn. contre qqn." [BK II 1075-6] ||| LECu.: Somali mād "protection", mād-iy- "to protect, safeguard" [LS] ||| NOm. *mād- "to help" [LS 1997, 456] ||| WCh.: perhaps Tangale madi "to protect, help, save" [Jng. 1991, 118].

NB: O.V. Stolbova $(1996,113)$ equated Tng. madi (semantically unconvincingly) with CCh.: Mafa meč- "donner tout ce que est dû à qqn.”.

484. AA * $\boldsymbol{m}$ - $\boldsymbol{d}$ "to reap" > SBrb.: EWlm. \& Ayr a-mad "1. cueillir (produits végétaux comestibles etc.), 2. ramasser (bois de chauffage; produit sauvage qqch.), 3. arracher, lever (racines, arachides, pommes de terre etc., à la moisson)" [PAM 2003, 520] ||| HECu.: Kmb. \& Sid. mid- "to cut crops, reap" [Hds. 1989, 46].

485. AA * $\boldsymbol{m}$ - $\boldsymbol{d}$ "(to accumulate in) mass of water" $>$ Ar. $m d d$ I "8. être en crue (se dit des eaux d'un fleuve), monter (de la mer, de la marée)", IV "être plein de sève, ètre juteux (se dit des plantes)", madd- "2. crue des eaux, 3. marée montante, flux de la mer" [BK II 1076-77] ||| NBrb.: Shilh ta-mda "lac, étang, mare" [Lst. 1942, 59, §148] | Mzg. mdu "1. stagner, croupir (eau), 2. être rempli d'eau (cuve, cuvette, puits, fossé etc.), a-mda $\sim a-n d a$ "étang, lac, mare", ta-mda ta-nda ta-nna "mare, flaque" [TAIFI 1991, 402-3], Izdeg a-mda "étang", ta-mda "marais, mare" [Mrc. 1937, 107, 161-2] | Iznasen \& Senhazha ta$m d a$ "flaque d'eau, trou profond dans un cours d'eau" [Rns. 1932, 385], Menaser ta-mda "(désigne) les mares d'eau restant dans le lit des fleuves quand ceux-ci sont desséchés" [Bst.] | Qbl. ta-mda ti-medwa "mare, réservoir, basin" [Dlt. 1982, 486], Bugi ta-mda “étang" [Bst.] || EBrb.: Gdm. te-mda "potager" [Bst.] = ta-māda, pl. t-madw-an "jardin de palmerie, irrigable" [LANFry 1973, 197, \#968] (Brb.: Bst. 1885, 166) ||| Bed. māda "deep water, sea" [Hds. 1996, 89] || LECu.: Orm. madd-ă "(last drop of) water", madd-isa "to drink up" [GRAGG 1982, 273] | HECu.: Burji madd-ō "spring", madd- "to spring (flow from spring)" [Sasse] = madd-ó "spring of water" [Hds. 1989, 141].

NB: E. Laoust (1.c.) derived the Brb. word (with prefix $m$-) from $\sqrt{ } d$, cf. Hgr. $\breve{e}-d u$ "mouiller, imprégner d'un liquide et être imbibe" [Lst.]. 
486. AA * $\boldsymbol{m}$ - $\boldsymbol{d}$ (perhaps * $\boldsymbol{m} \boldsymbol{u} \boldsymbol{d}-/ \boldsymbol{*}^{\boldsymbol{w}} \boldsymbol{a d}$-?) "groin" > ES (borrowed < Oromo): Wolane $m u$ damudo "groin" [Lsl.] ||| LECu.: Oromo mudāmudd̄̄ "groin" [GRAGG 1982, 292] | HECu.: Hadiya muday-iččo "groin" [Lsl.], Qabenna možožži-ta "groin" [Lsl.] (ECu.: Lsl. 1986, 378) ||| WCh.: AS * $m^{w} a t$ [- $t<*$ - $d \#$ reg.] (prefixed by *po-) "groin” [GT 2004, 259]: Angas po-mwat "groin" [Gochal 1994, app.], Mpn. mwát "private parts (male or female, not an obscene word)" [Frj. 1991, 39], Msr. po-mwat "cervix", sorop ku po-mwat "women have cervix" [Dkl. 1997 MS, 250]. Eventually related to AA *m- $t$ "waist" (below)?

487. AA * $\boldsymbol{m}$-t "to press" > Ar. matta V: tamattā "s' appuyer fortement sur un bout de la corde, voulant la casser" [BK II 1055] ||| LECu.: Orm.-Borana mit-ō "force, oppression”, mit-is/fa "to compel, force, press" [Strm. 1995, 210]. A var. root to AA *m-d "to press" (above).

488. AA *m-t (var. *m-t? ) "waist, hip" > Sem. *matn- [ext. *-n-?] "hip and small of the back" [SED I 173-4, \#192] ||| LECu.: Oromo mudd-i- [- $d$ - reg. <*-t-] "waist” [GRAGG 1982, 293; Hds. 1989, 163] ||| WCh.: perhaps SBch. * $m \bar{V} s_{-}$" "hip, waist" [GT]: Guruntum muusi [-s- <*-t- reg.] "hip" [JAGGAR 1989, 185], Guus \& Sigidi MiIs [- $s<*-t$ reg.] "waist" [CARON $2001,29]$. Eventually related to AA * $m$ - $d$ "groin" (above)?

NB: Cf. perhaps also WCh.: PAngas * $m$ wat "trunk, stem (of a tree)" [GT 2004, 259]?

489. AA * $\boldsymbol{m}$ - $\boldsymbol{t}$ "bone" > Bed. mita "Knochen" [Lmb. 1993, 352 with a false Cu. etymology] ||| WCh.: SBch. *mwat "bone" [GT]: Zaar mwat [IL apud JI 1994 II 36], Zaar of Gambar Leere mwàt [Smz.], Zaar of Lusa mwât [Smz.], Zakshi mwat [Smz.] (SBch.: Smz. 1978, 21).

490. AA * $\boldsymbol{m}$ - $\boldsymbol{t}$ "to eat" > NOm.: NWOmt. *mitt- "to swallow" [GT]: Wolayta \& Dawro \& Dache mitt- "to swallow" [LS 1997, 472], Gamu mitt- "to swallow" [SotTILE 1999, 439] ||| CCh.: Fali mitá "to swallow" [Ennulat $1976 \mathrm{MS}, 2$, \#40] | (?) Gisiga módì [- $d-<*-t-$ ?] "to ruminate" [Rsg. 1978, 318, \#600] | Masa müt-nà "manger (en mêchant)" [Ctc. 1978, 73] = "manger (grains)" [Jng. 1973 MS] = mút-nā "to eat (soft things)" [Jng. in JI 1994 II, 121], Ham \& Musey \& Lew \& Marba mút "mâcher, manger graines crues" [AJELlo 2001, 34-35]. An old var. root is attested in AA *m-t (below).

491. AA * $\boldsymbol{m}-\boldsymbol{t} \sim * \boldsymbol{m}-\boldsymbol{d}$ "to hit" > ES: Amh. mätta "to hit" [Hds. 1989, 80], cf. perhaps also Geez madada "1. to hit, 2. execute, 3. Spread" [Lsl. 1987, 329 with a diff. Sem. etymology] ||| NOm.: Nayi mútn- "to beat" [Bnd. 1996 MS, 3, \#43] ||| WCh.: PAngas * $m^{w} a t>* m a_{3} t$ (?) "to beat" [GT 2004, 259]: Angas muat "to beat, strike" [ORMSBY 1914, 208, 315] = mwat mwot $\sim$ mat "to beat, strike" [Flk. 1915, 250-251] = mwàt $\sim$ mwjt (K) "schlagen, hauen, to beat" [Jng. $1962 \mathrm{MS}, 27]=$ mot (sg.) "to hit" [ALC 1978, 39] = mwàt "to hit" [Krf.] = mwàt "to hit" [Gcl. 1994, 69, 120].

NB: P. Newman $(1970,42)$ equated Angas mwat with Hausa maar $-[-r-<*-d-]$ "to beat, slap".

492. AA * $\boldsymbol{m}$ - $\boldsymbol{t}$ "to wish": SBrb.: EWlm. \& Ayr madəmmud-ət "désirer ardemment", e-mădămmăd "désir" [PAM 1998, 210; 2003, 525] ||| HECu. *mit- "to wish (for)" [Hds. 1989, 169] || SCu. *mit- (?) [GT] > Irq. mits-imm "to long for" [MQK 2002, 74] ||| ECh.: Kwang mèdi "demander (qqch. à qqn.)" [Jng. 1973, 47], Kwang-Mobu mè:ḍ̂ "to request (demander)" [Jng. 1972 MS, 22, \#586] = módé “demander" [LENSSEN; Brt.-Jng. 1990, 148: <*m-d], Ngam mèdi “demander (qqch. à qqn.)” [Lenssen 1984, 69]| Birgit màadí “demander" [Jng. 1973 MS; 2004, 356].

NB: Perhaps remotely related to a better known (already published) AA root, namely AA * $m-[h]-d$ "to ask, beg" 
[GT]: Brb. *m-[h]-d "to beg" [GT]: EBrb.: Audjila $m \bar{u} d$ "pregare" [Prd. 1960, 172], Gdm. $m \bar{u} d$ "prier" [LANFRY 1973, 193-4, \#965] $\rightarrow$ a-mudu "prière" [Lst. 1931, 284] || SBrb. *muhed [Ksm.]: Hgr. muhad "prier, réciter" [Prs.], EWlm. mud "prier" [PAM 1998, 208], Ghat muhed "réciter en priant" [Fcd. 1951-2, 1156-7] = muhad

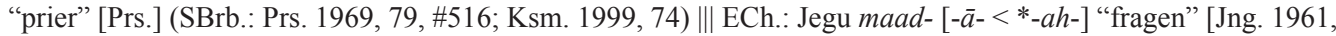
114]. Cf. also Ch. *?Vnd- "to ask" [GT]: WCh.: Bole ?und- "(an)rufen" [Lks. 1971, 133] || ECh.: WDng. índé "demander (?)" [FÉDRY; Brt.-Jng. 1990, 148: <*m-ḍ], EDng. inde "fragen" [Ebs. 1987, 82], Bdy. 9ìnde "demander" [Fédry] (DM: FÉDRY 1977, 107) <*H-m- $d$ [GT]? For the Brb.-Jegu comparison see HSED \#1788. Needless to say that this AA root has nothing to do with Eg. $m d w$ "sprechen, reden" (OK, Wb II 179) as suggested in IS 1976, \#311 \& HSED.

493. AA *m- $\boldsymbol{t}$ (var. *m-t?) "1. to be hidden, 2. disappear": HECu. *māt- "to hide (tr.)" $\rightarrow$ *māt-am- "to hide (intr.)" [Hds. 1989, 79] > i.a. Sidamo mat "nascondere" [Crl. 1938 II, 215] = mâta (sic, -t-) "to conceal, hide" [Gsp. 1983, 227] || SCu.: Ma'a -matu "to hide" [Ehret 1974 MS, 44; 1980, 156, \#29] ||| WCh.: Hausa mádè "to be extinguished" [Abr. 1962, 630] || CCh.: Margi mád (adv.) "hiding in the ground (?)" [Hfm. apud RK 1973, 125] | Uldeme -mámàd- “1. disparaître, périr, 2. se cacher” [Clm. 1997, 199] || ECh.: Kera mété "sich verstecken" [EBERT 1976, 80].

NB1: Ch. Ehret (1980 1.c.) combined the Ma'a verb with Rift *mah/h- "shadow" < SCu. *mah, which is a false etymology.

NB2: JI 1990 MS, 2, §33 explained ECh.: Sarwa mōsâ \& Gadang mós “cacher” from Ch. *m-t.

494. AA * $\boldsymbol{m}$ - $t$ "to eat" > NBrb.: Mzg. mdey "goûter (la nourriture)" [TAIFI 1991, 406], Izdeg $m d i$ "goûter" [Mrc. 1937, 130] | Mzab ə-mdi "1. goûter, 2. se rendre compte en goûtant" [Dlh. 1984, 116], Iznasen $t u-m d i^{k}-t$ "action de faire goûter qqch. à qqn." [Rns. 1932, 385] ||| HECu.: Sidamo mummûta "to chew, keep sg. sweet in one's mouth", muṭa mummuṭa "to suck (child)", mutti mutti assa "to suck a liquid" [Gsp. 1983, 241, 243] ||| WCh.: Hausa móódè "1. to gulp down all of liquid, 2. suck in (lips)" [Abr. 1962, 677]. A var. root is present in AA *m-t "to eat" (above).

495. AA * $\boldsymbol{m}$ - $t$ "1. sweet, 2. good" > Om. * $m A t$ "good" [Bnd. 1994, 1156, \#34] > i.a. Hozo matți [Bnd.] ||| WCh. *m-ḍ- "sweetness" [HARunA 1993, 78, \#44]: presumably Hausa mádi "a sweet drink made from juice of sugar-cane and various trees" [Abr. 1962, 630] | Grnt. mwadami "sweet" [Jgr. 1989, 188] || CCh.: Hurzo màdáy [Rsg. 1978, 339, \#712] || ECh.:

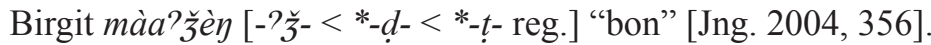

496. AA * $\boldsymbol{m}$ - $t$ (perhaps *mut, var. *m-t) "to change": NBrb.: Zayan \& Sgugu mutti "1. changer, 2. être versatile (individu), 3. se déplacer, décamper” [Lbg. 1924, 568] || WBrb.: Zenaga $\sqrt{ } m-t-k>$ a-mtettek "changement" [Bst. 1909, 241] || SBrb.: Hgr. mutti "1. être changé, 2. changer" [Fcd. 1951-2, 1259], EWlm. \& Ayr mătṭăy "être change" [PAM 2003, 567] || LECu.: Oromo mūta "1. to change (intr.), 2. shed skin (snake)" [GRAGG 1982, 296] $=m \bar{u} t \bar{a}$ "to change (intr.)" [Hds. 1989, 38] ||| CCh.: PMasa *mud > *mbud "to change" [GT]: Gizey/Wina mùd, Masa mù?[-?<*-d?], Ham \& Musey \& Lew \& Marba mbùt [-t<*-d?] "échanger, transformer" (Masa gr.: AJELLo 2001, 21, 56).

497. AA * $\boldsymbol{m}$ - $t$ " to become vigorous" > Ar. mata $\bar{a}$ "1. recueillir ses forces pour marcher rapdement" [BK II 1124] ||| SCu. *mat $t^{(y)}$ - [Ehr.]: Ma'a -ma'ai "to take up work with renewed vigor”| Dhl. mat- "to wake up" (SCu.: EHrET 1980, 153, \#4).

498. AA *m-t "to see" > Ar. $m \underline{t} w$ I "7. ouvrir les yeux" [BK II 1124] ||| WCh.: Kupto mèd"to see" [LEGER in JI 1994 I 145]. 
499. AA * $\boldsymbol{m}$ - $\boldsymbol{T}$ (perhaps * $\boldsymbol{m}$ - $\boldsymbol{t}$ ) "to pinch": LECu.: Saho \& Afar \& Somali $m \bar{u} d$ - | Oromo múta "stechen” (LECu.: Rn. 1886, 879) || SCu. *mut- "long narrow point” [Ehret 1980, 158] ||| CCh.: Masa mètá "to pinch" [Brt. 1995, 223].

500. AA * $\boldsymbol{m}-\boldsymbol{T}$ (vars. * $\boldsymbol{m}-\boldsymbol{d} \sim *^{\boldsymbol{m}} \boldsymbol{t} \boldsymbol{t}$ ) "part of the face: cheek, temple" > NBrb.: Mzg. $i$-mtittu "tempe" [T AIFI 1991, 445] || SBrb.: Hgr. é-med, pl. i-medd-en "tempe et région zigomatique: tempe et partie de la joue qui est immédiatement audessus de la tempe entre la pommette et l'oreille" [Fcd. 1950-1, 1163], EWlm. \& Ayr a-md̆d, pl. i-măḍ-ăn "tempe" [PAM 1998, 210] ||| LECu.: Oromo madd-ī "cheek" [GRAGG 1982, 273; Hds. 1989, 39], Oromo (Borana, Orma dials.) madd-i "the cheeks, temples" [Strm. 1987, 362] ||| CCh.: Muyang a-mid "chin" [Rsg. 1978, 225, \#131] < CCh. *m-d "chin" [JS 1981, 70B]. Irreg. alternation of -d- $\sim-d-$.

NB: The derivation of the Mzg. form < i-metti "larme" (Taifi 1.c.) may be rather merely a Volksetymologie.

501. AA * $\boldsymbol{m}$ - $\boldsymbol{T}$ (vars. * $\boldsymbol{m}-\boldsymbol{d} \sim * \boldsymbol{m}-\boldsymbol{t}$ ) "tears" > NBrb.: Shilh $\boldsymbol{a}$-mțta "larme" [Jst. 1914, 121] | Mzg. i-metți "pleur, larme" [TAIFI 1991, 445], Izdeg i-metți "larme" [Mrc. 1937, 153], Zayan \& Sgugu $i$-mti $\sim$ i-metți "larme" [Lbg. 1924, 568], Ait Ndir a-motța "tear (n.)" [Pnc. 1973, 107] | Nfs. i-mottt-aun (pl. of a-metța) "pleurs" [Lst.] etc. | Qbl. i-metți "larme" [Dlt. 1982, 527], Zwawa \& Bugi i-metti, pl. i-metta-un "larme" [Bst. 1890, 316] (NBrb.: Bst. 1890, 62-63; BiARNAY 1917, 90) || EBrb.: Gdm. Vm-t: a-motta, pl. matta-w-en "larme" [Lnf. 1973, 220, \#1049] || WBrb.: Zenaga $\sqrt{n-d}-w$ : $\dot{e}$-ndaw-ən (coll. pl.) "larmes" [Ncl. 1953, 227] || SBrb.: Hgr. ă-mit, pl. i-mett-aw-en "larme" [Fcd. 1951-2, 1163], EWlm. a-mott \& Ayr a-mott "larme" [PAM 1998, 228], Tadghaq \& Tudalt a-mott "tears" [SudLow 2001, 281] ||| HECu. *indìdd-o "tears of eyes" [Hds. 1989, 149] < *imdìdd-o [GT] ||| NOm.: PMaoid *? amt- (?) "tears" [GT] > Hozo ámt-i \& Sezo hamiç(l) "tear of eye" [Sbr.-Wdk. 1994, 17, \#25] || WCh.: Pero múddi "tear (lacrima)" [Frj. 1985, 42]. Irreg. alternation of $-d-\sim-d-$.

NB: The derivation < titt? "eye" (Taifi 1.c.) may be merely a Volksetymologie.

502. AA * $\boldsymbol{m}-\boldsymbol{T}$ (perhaps * $\boldsymbol{m} \boldsymbol{u} \boldsymbol{T}$-?) "sprout" > NBrb.: Shilh $\boldsymbol{a}$-mud "semence" [Jst. 1914, 121] | Izdeg a-mud, pl. i-madd-en "(graine de) semence” [Mrc. 1937, 130, 233] ||| HECu. *mutt-i? r- "to sprout" [Hds. 1989, 141] vs. Sid. mud-e "sprout" [Hds.] | Orm. mut-a "sprout, blade (crop)" [GRAGG 1982, 295] = mutt- $-\bar{e}$ "sprout" [Hds.].

NB: Cf. also SLECu. *mid- [Black]: Orm. míd-āni "grain" [Black] = mid-ān "grain (growing, harvested, or food)" [GraGg 1982, 285], Konso mid-āa \& Gdl. mičca "edible leaves" [Black], Som. mídó "fruit" [Abr. 1964, 179] (LECu.: BlACK 1974, 218) | HECu.: Gedeo (Drs.) mid-a mi?r-a "grain, cereal, crop" [Hds. 1989, 72]. Irreg. alternation $-d-\sim-d-$. From AA $* m-d \sim * m-t$ ?

503. AA * $\boldsymbol{m}-\boldsymbol{t}-\hat{\boldsymbol{s}}$ "to separate" > Ar. mataša "séparer et disperser qqch. en passant ses doigts dans qqch." [BK II 1056] ||| CCh.: Mafa mutus - (-sl-) "arracher (qqch. qui est retenu par un fil)", mutus- "(dé)couper, arracher” [Brt.-Bléis 1990, 234].

504. AA * $\boldsymbol{m}-\boldsymbol{t}-\boldsymbol{k} \rightarrow$ assim. * $\boldsymbol{m}$ - $\boldsymbol{t}-\boldsymbol{k}$ "to be sweet, sticky (of ripe figs)": Sem. *mtq "to be sweet": Akk. matāqu "süß sein, werden" [AHW 632] || Hbr. mtq qal "2. süß sein", mātōq "süß” [GB], Aram. mtq "(mit Wohlgefallen) saugen” [GB] || Ar. $m t q$ [-t- $<*$ *t- before -q-] V "mit Wohlgefallen schnalzen, schmatzen", maț-at- "Süßes, Honig" [GB] (Sem.: GB 475)

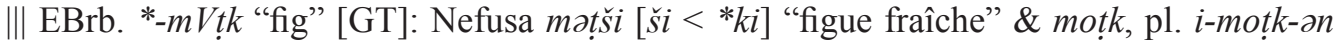
"figue" [Lst.] = motk, pl. i-mótk-ån [irreg. -k-] "fico fresco" [Bgn. 1942, 305], Siwa $i$-moț̌an (pl.) "figues" [Lst.], Sokna $a$-məčč "figue" [Lst.], Sened $a$-mot ši "figue" [Lst.] (EBrb.: Lst. 1931, 238) ||| LECu.: presumably Oromo muduqa "overripe, soft", muḍq క̌eda "to be 
very rotten (e.g. banana)" [GRAGG 1982, 293] ||| CCh.: Glavda mtaka "sweet" [RB 1968, 66] || ECh.: Mokilko mádidàk máddàk "sucré”, mádidiká “sucrérie, saveur” [Jng. 1990, 136], WDng. mùdùk mùdùk mìmùdùk "collant" [FÉDRY 1971, 138].

NB: The Sem. root has been often used in Nst. (IE-AA) comparisons, but its Berber and Chadic cognates have been - to the best of my knowledge - up to now neglected. Only the HSED \#1818 has mentioned a comparison between Sem * $m t q$ and a certain Matakam mtake "sweet", which is however not found in Rsg. 1978, \#211-212!

505. AA * $\boldsymbol{m}$-t- $\dot{\boldsymbol{q}}$ "to eat" > Ar. mataha "1. lécher (le miel, etc.), 2. manger beaucoup" [BK II 1121] ||| SBrb.: Ayr mataq (interj.) "son de mastication silencieuse" [PAM 2003, 564] ||| CCh.: Bachama midúks [met. <*m-t-k] "schlucken" [Skn.].

506. AA * $\boldsymbol{m}-\boldsymbol{t}-\boldsymbol{h}$ "to pour out, emit fluid" > Ar. mataha "1. tirer de l'eau (d'un puits), 2. rendre les excréments, 3. peter, lâcher un pet” [BK II 1056] ||| SCu. *matah- [Ehr.] > Dhl. mantah- "to vomit", mantah-aðēð- "to purge" [EHRET 1980, 323, \#30 with a false Som. etymology].

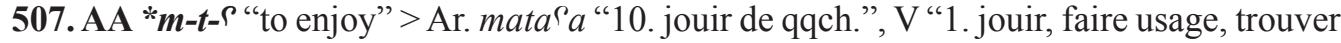

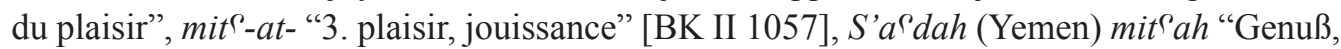
Vergnügen" [BEhnstedt 1987, 301] ||| SCu.: Dhl mutuৎ-uð- "to masturbate” [EEN 1989, 38; EHRET 1980, 159, \#52 with a false SCu. etymology].

508. AA * $\boldsymbol{m}$-T-r "shoulderblade (?)": Eg. mjd3 [<*mydr or *mytr] "ein essbarer Körperteil des Rindes (vom Bein?)" (PT, Wb II 45, 7) = "upper foreleg, lower foreleg" (AEPT ad PT $1546)$ = "ein Körperteil vom Rind (als Speise)" (GHWb 326) ||| ES: Geez mațarā mațāāa "shoulder(blade)", cf. Tigre materär "spine" (ES: Lsl. 1987, 373; cf. Rn. 1887, 278).

NB: EBrb.: Gdm. $\underline{u}$-madir, pl. midar "1. omoplate, 2. houe large à manche court qui forme avec le plan de l'outil un angle très fermé" [LANFrY 1973, 206, \#978] is certainly out of question, since it is cognate with NBrb.: Shilh a-madir "Jäthacke" [Stumme] = "1. Haue, Hacke, 2. Stangengebiß des Pferdes" [Vcl.], Wargla a-mdir, pl. i-midar "1. sorte de houe, de sape, 2. par ext. omoplate" [Dlh. 1987, 185], Qbl. a-mder, pl. i-medr-an "1. rebord d'une porte (seuil), d'une fênêtre, d'un bassin, 2. bois de charpentre, poutre" [Dlt. 1982, 487] || EBrb.: Gdm. u-madir, pl. midar "1. omoplate, 2. houe large à manche court qui forme avec le plan de l'outil un angle très fermé" [LANFRY 1973, 206, \#978] = o-madir, pl. midar "1. breite Hacke mit kurzem Stiel, 2. Schulterblatt (wegen der Hakenform)" [Vcl.] || WBrb.: Zng. $\underline{a}-m d \partial r \sim \underline{e}-m d \partial r$, pl. a-mdurūn "vers, du côté de..." [Ncl. 1953, 207], which are explained by Stumme $(1912$; cf. also ZA 27, 125) and Vycichl $(2005,4)$ from an unattested Phn. * $m$ ` $d r$ "Haue, Hacke" via Punic, cf. also Hbr. $m a^{\Upsilon}$ dèr "plough" [KB 609] = "1. (Jät)Hacke, 2. Pflugschar" [Vcl.].

509. AA *m-t-r "to cut, split" > SBrb.: EWlm. a-mdur \& Ayr a-ndu/ar "déchirure, fente, fêlure" [PAM 2003, 525] ||| HECu. *matar- "to cut branches, prune" [GT after Hds. 1989, 46].

510. AA *m-t-r "to fill up" > Ar. matara I "2. combler qqn. de biens, 3. remplir (une outre)" [BK II 1122] ||| NBrb.: Mzg. a-mettar "tas, amas, monceau” [TAIFI 1991, 445].

511. AA * $\boldsymbol{m}$-z (perhaps *miz-?) "right (hand)" > ECu. *mizg- (root ext. *-g?) "right side" [SASSE 1979, 25, 57-58] ||| NOm.: Male mizaki "right (side)" [Sbr. 1994-5, 10] || SOm.: Ari mizi "right (hand)" [Bnd. \& Tully], Galila mīži [Flm. apud Bnd. 1994, 157] (SOm.: Bnd. 2003, 350, \#72) ||| ECh. *m- $d$ [*-d < AA *-3?] "right" [GT]: Barein medu "rechts" [Lks. 1937, 51] | WDng. (Karbo) meedênn "rechts" [Lks. 1937, 17] = mecdén [Gouffé] = mèèdà “(à) droite” [FÉDRY 1971, 127], EDng. mèedā “à droite” [Dbr.-Mnt. 1973, 200] | Mokilko 
?àmmádè "la droite", mádiká "le côté droit, la droite" [Jng. 1990, 58, 136] | Toram mìde "droite" [Alio 2004, 260, \#317].

NB: C. GoufFé $(1971-2,113, \S 7)$ equated the Dangla root (via met.) with Hausa daama "droite" $<* \sqrt{ } m-d \sim * \sqrt{ } d-m$.

512. AA *m-s "to wipe, rub" > Sem.: JNAram. mšy "to wipe, clean" [SABAR 2002, 226] $\|$ Mhr. môsī "reiben" [Müller, not in Jns. 1987] || ES *mss "to rub" [GT] vs. Grg. mwašä "to rub" [Lsl. 1979 III, 432-3] (ES: Lsl. 1982, 54; Sem.: MüLler 1981, 401) ||| NBrb.: Mzg. a-mes "1. enduire, 2. oindre, 3. frotter une chose contre une autre" [TAIFI 1991, 435], Izdeg a-ms "enduire" [Mrc. 1937, 34], Zayan \& Sgugu a-mes "frotter une chose contre une autre" [Lbg. 1924, 567] | Mzab a-mos "masser, essuyer fortement, frotter" [Dlh. 1984, 122], Wargla $a$-məs "frotter, essuyer fort, masser" [Dlh. 1987, 197] || SBrb.: Ghat a-mes "effacer, ôter l'empreinte" [Nhl. 1909, 154], EWlm. e-mmas “effacer, gratter, décolorer" [Ncl. 1957, 571] = EWlm. \& Ayr a-mas "essuyer, effacer" [PAM 2003, 555] ||| LECu.: Afar musu $e$ [ext. "to be peeled, scraped sore" [PH 1985, 172] | HECu.: Sid. miššiga [ext. -g] "to rub with the hands" [Gsp. 1983, 234] ||| CCh.: Lamang mas-a [mósó] "einreiben" [Wolff 1972, 198] | Glavda mas "to anoint" [RB 1968, 63]. Cf. also Sem. *mḩ̌̌ [ext. *-h-] "to rub" [GT] > Ar. mahasa "frotter avec force" [BK II 1066] || Tigre mähasa "to wipe" [LH].

NB: C. Brockelmann $(1932,811)$ affiliated Brb. *a-mes "frotter" [Bst.] with Ar. massa "toucher" [BK II 1101] = "berühren" [Brk.], which is semantically dubious.

513. AA *m-s "fire" > Ar. $m \bar{a} m \bar{u} s-$ "feu" [BK II 1154] ||| Brb. *ti-mVsi "fire" [Mlt.] = *ti-msi [GT]: e.g. NBrb.: Mzab ti-msi "feu" [Dlh. 1984, 123] Q Qbl. ti-mess ti-mes ti-messi $t i-m s i$ "feu, l'enfer" [Dlt. 1982, 520] || EBrb.: Siwa to-msi \& Sokna ti-msi "feu" [Lst. 1931,

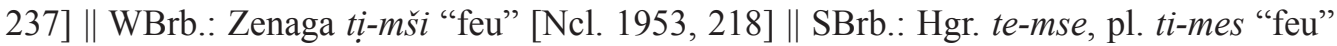
[Fcd. 1951-2, 1247], EWlm. \& Ayr te-mse "1. feu, 2. Lumière" [PAM 1998, 223] ||| CCh.: Daba $m s a$ "to roast, grill" [Pascal] = mis $\bar{a}$ [LIENHARD in JI 1994 II 275] = màsā "to roast" [Brt. 1995, 226] || ECh.: perhaps Kera ká-msí "ashes and water" [PEARCE 1998-99, 67]. NB1: Cf. perhaps also NOm. *mič- $(<* m i \check{z}-$ ?) [GT]: Wolayta mičč- "to taste hot" [Hyw.], Zayse mičč- "to roast" [Hyw.], Koyra mičč-e "heiß" [Lmb.], Baditu mičč-e "caldo" [Crl. 1929, 62], Haruro meç-āys "scaldare, bruciare", mičçc-e "caldo" [CR 1937, 653] | Gimirra-Benesho mič- "to roast" [Hyw.] | Chara miča "to burn" [Bnd. 1974, 29] | Kaffa mī̌̆- "heiß sein, brennen", mî́ž-o "Hitze" [Rn. 1888, 316] = mič "1. accendersi, 2. Arrostirsi" [Crl. 1951, 468] = mičč "Feuer fangen, gebraten werden, verbrennen (intr.)" [Lmb.], Mocha mičç̌ci(yé) "to burn (intr.)", mičči (yé) "to burn, fry, kindle" [Lsl. 1959, 39-40] = mičč- "to roast" [Hyw.], Sns.-Bworo micc- (-tts-) "1. (an) brennen (tr.), 2. verbrennen (tr.), 3. Frittieren" [Lmb. 1993, 361] | Sheko mičč- "1. anzünden, 2. verbrennen (tr.), 3. Frittieren", mičč-o "heiß” [Lmb.] (NOm.: Hyw. 1988, 283) ||| CCh.: Glavda mč- "verbrennen" [Rapp in JI 1994 II 55], although the sibilant correspondence is not clear.

NB2: A. Ju. Militarev (1991, 259, \#28.1) prefers comparing the common Brb. root "fire" i.a. with Sem. Ar. mays-ān-, pl. mayāsīnu "étoile qui brille d'un vif éclat" [BK II 1173], Sem. *9amš- "yesternight" [GT] and even Eg.-Sem. *m-s "night" [GT]. Semantically unconvincing.

514. AA *m-s "back, haunch" (perhaps *mas-?): NBrb.: Qbl. a-mmas "les hanches et le bas du dos" [Dlt. 1982, 481] ||| Ch. *m-s "back" [JS 1981, 32]: WCh.: Fyer \& Tambas \& Bokkos más "Rücken" [Jng. 1970, 88, 144] || CCh.: Hina mesé "Gesäß” [Str. 1922-23, 116] || ECh.: EDng. máasá "rein, région médiane du dos" [Dbr.-Mnt. 1973, 197], WDng. mààsò "région médiane du dos" [FÉDRY 1971, 123], Bdy. maasi "hanche, basin" [AJ 1989, 96]. Cf. AA *m-s-k "back" (below).

515. AA * $\boldsymbol{m}-\boldsymbol{s}$ "middle" > presumably Ar. mas? - "milieu de la route" [BK II 1102] ||| Common Brb. *a-mmas "middle” [GT]: e.g. NBrb.: Qbl. i-mass-en (pl. only) "1. l'intérieur du 
pain, de la galette, mie de pain" [Dlt. 1982, 520] || EBrb.: Gdm. a-mmas "milieu, centre" [LANFry 1973, 218, \#1032], Siwa a-mmās "centre, milieu" [Lst. 1931, 209, 260] || WBrb.: Zenaga $a^{\dot{\delta}}$-mməš $\sim a^{u}-m m ə \check{z} \sim a^{u}$-mmaš "milieu”, $\dot{o}-m m ə \check{s}$ "milieu, fond, dessus" [Ncl. 1953, 217] || SBrb.: Hgr. â-mmas "intérieur, la partie intérieure” [Fcd. 1951-2, 1246], EWlm. \& Ayr a-ṃmas "milieu, centre, intérieur" [PAM 1998, 224] (Brb.: Bst. 1890, 68, 315; 1909, 245) ||| WCh.: AS *m $\breve{\bar{s}}$ "inner part of plants" [GT 2004, 247]: Angas mees "pith (of guinea corn stalks, grass, etc.)" [Flk. 1915, 244], Gmy. mès [- $\left.\varepsilon_{-}^{-}\right]$"the hard inner part of a tree, the heart of a tree" [Srl. 1937, 138].

516. AA *m-s "to kiss": MSA *msy: Hrs. amēs "to kiss the nose, greet" [Jns.], Mhr. amōsi [Jns.] = mōsī [Jahn] "to kiss (tr.)" (MSA: Jns. 1987, 272) ||| NBrb.: (?) Iznasen a-meḥhǎ́ [infix - h-?] "baiser voluptueux" [Rns. 1932, 389: not Arabism] ||| SCu.: Irq. ma? ás "to kiss" [Mgw. 1989, 115].

NB: Perhaps just an areal parallel (for the problem of MSA-Cu. contacts see BELova 1988 \& 1996)?

517. AA * $\boldsymbol{m}-\boldsymbol{s} \sim *^{\boldsymbol{m}}-\boldsymbol{s}-\boldsymbol{Q}$ (perhaps AA root extension *- $\dot{\text { ) }}$ " to smile": HECu. *mušik $y$ - "to smile" [Hds.] = *mišik - \& *mušš- (two contaminated HECu. roots?) [GT]: Gedeo mūš hiyy-, Hadiyya mišik y-, Kambata mušuk y- [<*mišik-?], Sidamo mušš y-, muši y- (HECu.: Hds. 1989, 137) || SCu.: Irq. misq-is- [Ehret] = misq-ís- "to smile" [Mgw. 1989, 115] || WCh.: Hausa mùrmùšíi [JI 1994 I, 108: < *mus-mus-, GT: semi-redupl. with the typical inetymological - $r$ - " "smiling, a smile" [Abr. 1962, 686] || CCh. *m-s "to laugh" [GT]: Bura muša miša "to smile, begin to laugh" | Bata \& Nzangi mos, mos "rire" | Daba mbis $\left[m b-<*^{*}\right.$ "rire", Musgoy mbis "rire" | Gidar mas, mas "rire" | Mboku mbass, Hurzo muš, Uldeme (Uzlam) mas (CCh.: BED 1953, 145; Mch. 1950, 37; 1953, 172; 1966, 136).

NB1: I cannot accept the SCu. etymology (*măk- "to be happy") offered for Irq. misq- by Ch. EHRET $(1980,155, \# 21)$. NB2: This AA root has perhaps Sem. reflexes too: cf. Geez mazha mazha [irreg. -z-] "to laugh (at), jeer at, dally (with women), be pampered", cf. also Ar. mazaha "to joke, jest" (Sem.: Lsl. 1987, 378).

NB3: Perhaps LEg. $m s \underline{h} \sim m s h 3 \sim m s \underline{h} \sim m s g$ (often [mis?]read as msh3) "1. intr. sich freuen (über etwas), 2 tr. (das Herz) erfreuen" (GR, Wb II 147) also belongs to AA * $m-s-\dot{q}$, but its etymology is still debatable: (1) Acc. to A.G. Belova $(1987,277)$, it is an $m$ - prefix reflex of Ar. sahira "(ver)spotten, sich lustig machen, verhöhnen, verlachen" mashara "lächerlich machen, verspotten, verlachen" [WeHR 1952, 365 \& 809]. (2) If the reading of the orig. root as $m s h 3$ is correct, cp. SBrb.: EWlm. \& Ayr măžgăl "être joli" [PAM 1998, 232]. (3) If $m s h$ was the root, cf. Akk. (jB) mašăhnu "etwa: aufleuchten (Stern, Meteor)" with the semantic shift "to shine" $\rightarrow$ "be happy", quite frequent in Sem.

NB4: I am not perfectly convinced whether PCh. ${ }^{*} g-m-s_{2}$ "to laugh" [JI $]$ would be the same root as AA * $m-s-[\dot{q}]$ and the CCh. * $m$-s would belong directly to it (as suggested in JI 1994 I, 108).

518. AA *m-s “(to) rain” > MSA: Jbl. musá?, Sqt. mássa "rain” (MSA: NAKANo 1986, 104, \#729) ||| HECu.: Burji mís-i (m) "heavy rain, cloud-burst”" [SASSE 1982, 145: isolated in HECu.] ||| NOm.: Anfillo amicc-o (-tts-) "rain" [Bnd.] | Hozo Imsi "rain" [Bnd. 1994, 1159, \#63] = Hozo \& Sezo imsi "rain" [Bnd. 1990, 609, \#63] = Sezo ?íns(?)i ?insi "rain" [Sbr.-Wdk. 1994, 15, \#223] ||| CCh.: cf. Bata myèsö "to splash, sprinkle (esp. water), dowse" [Pweddon 2000, 58-59] | Makeri \& Gulfei amesa "pluie” [Lbf. 1942, 169] || ECh.: Mokilko mízzi [irreg. -zz-] (pl.) "Regen” [Lks. 1977, 222].

Lit.: the Burji-NOm.-Kotoko-Mokilko paralle was first observed by H.G. MuKarovsky (1987, 178, 290-291; 1989 MS, 23, \#51). 
519. AA * $\boldsymbol{m}$-s "to fade" > Ar. msy: masā I "3. amaigrir, exténuer etc. (se dit de la chaleur qui fait maigrir les bestiaux)" [BK II 1108] ||| NBrb.: Mzg. msus "être fade, insipide" [TAїFI $1991,435]$.

520. AA * $\boldsymbol{m}-\boldsymbol{s}-\boldsymbol{k}$ "to get accustomed (?)" > SBrb.: Ayr $ə$-msək "être habitué à qqch. de bon" [PAM 2003, 558] ||| ECh.: Bdy. musàk"s'habituer, s'accoutumer" [AJ 1989, 101].

521. AA * $\boldsymbol{m}$-s- $\boldsymbol{k}$ "back of the body" > ES: Harari miskät "buttocks, anus" [Lsl. 1963, 112], Gurage: Selti \& Wolane \& Zway maskät "back of body, clitoris, female genital organs, *buttocks, anus" [Lsl. 1979 III 428] = Wolane maskät \& Zway maskit "back of body" vs. Selti məskät "female genital organ" [Lsl. 1963] ||| LECu.: Somali misikti "hip" [Lsl.] ||| CCh.: Kotoko mùskù "anus" [Bouny 1975, 4, \#34]. Cf. AA *m-s "back" (above)?

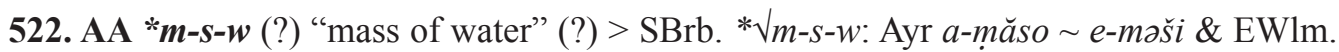
e-məšwi "puits, point d'eau” [PAM 1998, 557] ||| CCh.: Gsg. mesaúú, Balda măsaua, Holma mačoắ "Bach, Fluß” (CCh.: Str. 1922-23, 126).

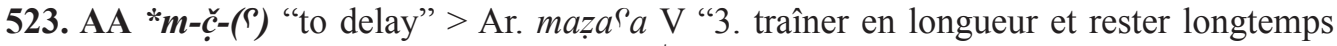
à faire qqch." [BK II 1125] ||| NBrb.: Mzg. $\sqrt{ } m-z$ : mmuz "être tardif (culture, fruit, labour), être dernier-né, naître tardivement" [TAIFI 1991, 450].

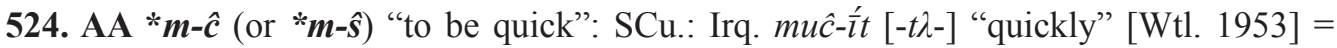

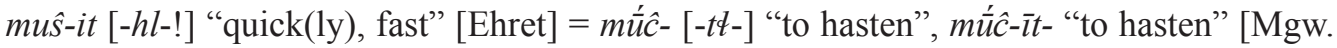
1989, 115] | Qwd. miŝmisia [-hl-] "fast" [corrected from Kohl-Larsen mizamiza by EHRET 1980 MS, 4] (Rift: Ehret 1980, 160, \#56) ||| WCh.: Hausa mázá “quickly” [Abr. 1962,

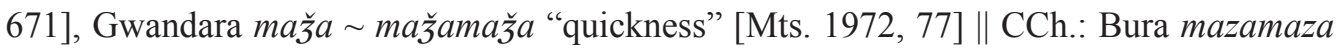
“quickly” [BED 1953, 131] | Hide msimsim "vite!” [EGUCHI 1971, 220].

NB: The distinction between $\mathrm{SCu} .{ }^{*} \hat{s}$ - and ${ }^{*} \hat{c}$ - was preserved in Qwadza in initial position (Qwd. $\hat{s}$ - and $\hat{c}$-, resp.), although I have no data proving the same in medial position (TAKÁCS 1999, 411). Therefore the $-\hat{s}-$ of Qwadza mišamis $a$ is not an absolute proof and support for the $\mathrm{SCu} .{ }_{-}^{*} \hat{s}$ - and Ehret's Irq. $-h l-=-\hat{s}$ - in this root (contra the $-\hat{c}-=-t \lambda-\&-t d-$ of Whiteley \& Maghway, resp.).

525. AA * $\boldsymbol{m}$ - $\hat{\boldsymbol{c}}$ "fat" > LECu.: Afar màs-u "fat" [Hyw. 1997, 110] | HECu.: Kambatta mešèla "fat (n.)", mešēl- "to be fat (of animal)", Sidamo mis- "to be fat (of human)" (HECu.: Hds. 1989, 61-62) ||| WCh.: Warji màch́yà-nà (-tt-) “oil”” [IL in JI 1994 II 260] || CCh.: Daba msáá "Fett" [Str. 1910, 461].

526. AA * $\boldsymbol{m}$ - $\hat{c}$ "to hurt, vex" (or sim.) > Ar. madda "1. faire souffrir qqn., 2. affecter qqn., lui causer de la peine, 3. faire du mal, piquer, picoter" [BK II 1118] ||| SBrb.: Ayr a-məd "nourrir de la rancune contre, être fâché contre", caus. šumad "vexer, fâcher, agacer, irriter" [PAM 2003, 524] ||| NOm.: Yemsa me?- [-?- <*-ç- reg.] "beleidigen” [Lmb. 1993, 362].

527. AA * $\boldsymbol{m}$ - $\hat{c}$ "to take, pick up" > Ar. $m d y:$ mad̄a "2. emmener, emporter" [BK II 1120] ||| Cu.-Om. *mad- "to pick up" [Lmb.] = *ma[ç]- [GT] > HECu. *mat- "to pick up" [GT] III NOm.: Gamu maç- (-ts'-) “sammeln, ernten, aufheben”, Dache \& Zayse maç- (-ts'-) “auf-

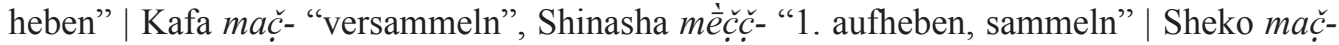
“sammeln, aufheben" (NOm.: Lmb. 1993, 106; 1993, 349; LS 1997, 475).

NB: Cf. also Ar. mașaha "7. ôter, enlever, emporter qqch." [BK II 1115] < var. AA *m-c? ?hether Brb. * $m-z$ (or *m-z?) "prendre" [GT] also belongs here is obscure (cf. alternatively AA *m-). 
528. AA * $\boldsymbol{m}$ - $\hat{c}$ (var. *m-c) "to wash" > Sem. *mș́mṣ́ *mșms: Ar. madmada "rincer la bouche ou rincer un vase avec beaucoup d'eau en l'agitant dedans" mașmașa "1. rincer légèrement (une vase), 2. se rincer (la bouche)" [BK II 1120, 1148], Dathina madmad "to rinse" [Lsl.] || Tigre mäșmäșa "to rinse" [LH] ||| PCu.-Om. *mAyAç(ç)- "to wash" [Dlg.]: ECu. *mayç- "to wash" [Dlg. 1983, 140]: LECu.: Somali-Isaq máyd-ayya "to wash" [Abr. 1964, 176], Oromo mičč- [Thiene, Bell] = mīç- "to wash clothes" [SASSE 1979, 26] = miččč-a "to wash (clothes, objects)" [GRAGG 1982, 285], Oromo-Borana mīč- [Andrzejewski], Konso mič- [Lmb.] | HECu.: Sidamo maço = mēčč- [Lmb.], Qabenna \& Alaba meçç̌i- [Lsl.], Kambatta mečçe? [Lsl.] = mečḉ- "to wash" [Hds.] (HECu.: Hds. 1989, 164) ||| NOm. *maydy- "to wash" [Lmb.]: Wolayta \& Dache \& Gamu mēçç-, Gofa meč- | Yemsa (Janjero) me?- [-?- < *-d-] | Kafa māčč-, Shinasha maš- | Sheko mass- (NOm.: Lmb. 1993, 93; 1993, 358, 362; LS 1997, 454; Cu.-Om.: Dlg. 1973, 308, cf. also Crl. 1951, 467; Lsl. 1959, 42), cf. Ongota mučmmuč-ada "swished water around in mouth, rinsed mouth out" [Flm. 1992, 193, 212].

NB1: The Cu.-Om. root has been usually (Rn. 1902, 307; Chn. 1947, \#487; IS 1976, 71, \#304; Dlg. 1970, 620, \#9; 1983, 140; 1992 MS, 83, \#178; Orel 1995, 108, \#113; HSED \#1748) compared with Ar. mwș I "1. frotter qqch. avec la main pour nettoyer, 2. Laver" [BK II 1168] (whose basic mng. may be different, cf. Lsl. 1987, 375) as well as Eg. $n \underline{d}$ "to grind" (which is both semantically and phonologically false).

NB2: This root should be distinguished from AA * $m-w-\hat{s}$ "to wash" (below).

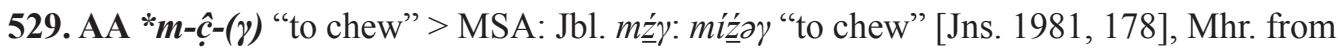

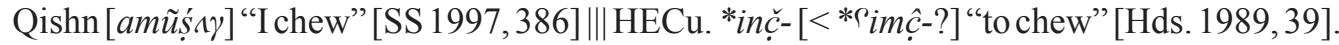

530. AA * $\boldsymbol{m}-\hat{\boldsymbol{s}}$ " 1 . to decay (naturally), 2. corpse" > Sem.: presumably Samar. Aram. msy [-s- $<*_{-} \hat{s}-$ reg.] qal "to decay" [TAL 2000, 479] ||| Bed. mi/esis "to die a natural death (animal), become carrion", mesūs-ánaí "carrion" [Rpr. 1928, 218] = mesūsanai "carcase" [Hds. 1996, 96] || LECu.: Afar maৎàsi [affix - - ] "carrion, carcase” [PH 1985, 158] || SCu.: Dhl. $m i \hat{s}-o(-\not-)$ "body of living person (as opposed to corpse)" [EHRET 1980, 160] $=m i \hat{s}-o(-h l-)$ "living body" [EEN 1989, 38] ||| NOm.: Mocha me.š-o "carrion" [Lsl. 1959, 42 with an unconvincing ES etymology] ||| WCh.: Hausa múúšè [hardl < *mūte] "corpse” [Abr. 1962, 688] | Boghom masáw “corpse" [Smz. 1975, 28; JI 1994 II 86] || CCh.: BM *m(a)ši "corpse" [GT]: Bura mši, Margi məši i, Chibak sai [<*msai] (BM: Hfm. 1987, 473, \#39).

531. AA * $\boldsymbol{m}-\boldsymbol{Z}$ "to press": Sem.: Akk. $m z$ ? or $m z y:(\mathrm{a} / \mathrm{mA}, \mathrm{jB}) m a z \bar{a}$ ? $u \rightarrow$ mazh "auspres-

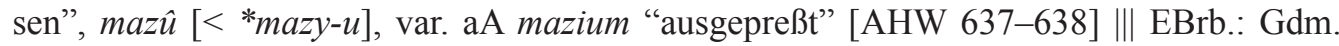
mimzz "être pincé, serré, 2. par ext. être dans la gêne” [LANFRY 1973, 222, \#1055].

NB1: In principle, LECu. *m- $d$ "to (op)press" (etymologized above from AA *m- $d$ ) could reflect ECu. *mV[z](no inner ECu. proof for $* z$ ), since AA * $z^{*} * \tilde{z}=\mathrm{ECu}$. $* z$, which regularly becomes * $d$ in LECu. Cf. LECu. * $m-d$ "to (op)press" [GT]: Oromo mudda "to press, tighten, torment" [GRAGG 1982, 292] | Rendille a-mída "ich belästige, bedränge, dominiere mit Gewalt" [SCHLEe 1978, 140, \#773].

NB2: The traditional Sem. etymology of the Akk. root is not impossible, cf. Ar. mzz "saugen", Mhr. muzz "to suck" (Ar.-Akk. suggested e.g. by von Soden \& LesLau 1987, 371). In this case, the primary sense might have been * "to press with mouth". For further eventual Sem. and AA parallels of Akk. mzy see Djk. 1981, 43, fn. 52.

532. AA * $\boldsymbol{m}-\boldsymbol{Z}$ "to wound" > presumably LEg. * $m z$ (?), written $m 3 z$ "verwunden, schlachten" (GR, Wb II 32, 1) $\rightarrow$ OCpt. MC- "verwunden" (KHW 520) ||| HECu. *maz- "to wound" [Lsl.] $=*_{m a d z-}$ [Hds.] \& HECu. *maz-a "wound" [Lsl.] = *madz-a [Hds.] (HECu.: Lsl. 1980, 125; Ss. 1982, 138; Hds. 1989, 170, 417)| LECu.: Boni mád-a? [-d-reg. < *-z-] "Wunde" [HeINE 1977, 294] ||| NOm. *maZ- [GT]: Kaffa may-o [-y- reg. < *-Z-] "wound" 
| Dizi: Sheko māy-o [-y-<*-Z-?] "wound” (NOm.-HECu.: Lmb. 1993, 100) || SOm.: Ari $m \bar{a} d-a\left[-d-<*_{-z-?]}\right.$ "scar" [Bnd. 1994, 157].

NB: The reading $(m 3 z, m 3 s, m z, m s)$ and the etymology of LEg. $m(3) z$ is disputable due to the late attestation, but this is probably not a late denom. of OEg. $m 3 z$ "knife".

533. AA * $\boldsymbol{m}$ - $\boldsymbol{Z}$ (act. *miZ-) "sort of cattle": Eg. mjz.t "animaux à cornes (?)" (CT I 289c, AL 78.1667) = “*Horntiere" (GHWb 326) ||| NOm. *miz- "bue, vacca” [Crl.] (NOm.: Rn. 1888, 318; Crl. 1938 III, 80, 115, 173, 206; 1951, 471-3; Lmb. 1993, 100).

534. AA * $\boldsymbol{m}-\boldsymbol{Z}$ "to take" > Eg. $m z$ "herbeibringen" (OK, Wb II 135, 7) ||| perhaps Common Brb. *m-z (or *m-z?) "prendre” [GT] ||| ECh.: Kwang-Mobu mǎ̌e "empoigner” [Lenssen 1982, 19], Ngam mači "poignarder” [Lenssen 1984, 9] | Tumak mùží "voleur” [Cpr. 1975, 84] (ECh.: Stl. 1996, 82 with a different AA etymology).

535. AA *m-S "joint of body" > NBrb.: Zemmur ta-měšaš- $t$ "rotule" [Lst. 1918, 4], cf. also Qbl. a-mě̌šă "fesse” [Dlt. 1982, 520] ||| LECu.: Afar māsayya (f) "joint of body" [PH $1985,158]$.

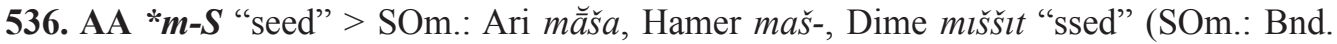
1994, 157) ||| WCh.: Daffo \& Bokkos masút "seed” [Jng. in JI 1994 II 286].

537. AA *m-g "dark" > SBrb.: Ayr măg-ăt măgăy "avoir les lèvres noires, être noir (bouche d'animal)" [PAM 2003, 526, 528] ||| LECu.: PSam *múgdi [root ext. *-d-?] "darkness" [HeIne 1978, 69/91] = Omo-Tana *mugd- "Dunkelheit" [SAsse 1981, 155] ||| ECh.: Kwang màgùwà (f) “soir” [Jng. 1973, 44] | EDng. màakò \& WDng. mààkó (f) "le soir, crepuscule" [Dbr.-Mnt. 1973, 192]. The ECu. root was affiliated by H.-J. Sasse (1.c.) with Kuliak: Ik moku "Nacht".

538. AA * $\boldsymbol{m - g}$ "to pour" > Ar. $m \breve{g} \breve{g}$ "jeter dehors, hors de la bouche (p.ex. salive ou un peu de vin qu'on avait pris dans la bouche)", IV “3. être en sève (se dit des arbres)", VII "couler tomber de la plume (sit dit d'une goutte d'encre qui s'échappe de la plume)", muğğ- "goutte (p.ex. de miel qui tombe par terre)", muğăğg- "1. salive, bave qui coule sur les coins de la bouche, 2. Miel" [BK II 1062-3] ||| ECh. *ma/ug'- "to flow out" [Ehr.]: Somali magay"hole in ground where water stagnates" [Ehr.] | Dullay: Harso \& Dobase mag- "ausgießen" [AMS 1980, 174], Tsamay $m \bar{e} g$ "to pour" [SAVÀ 2005 MS, 262] | Yaaku -mug-ur- "to leak" [Heine] (ECu.: EHret 1991, 244, \#143).

539. AA *m-[g] "man" > ECu.: Yaaku məgəi məqəi "man (male)" [HeINE 1975, 129] = "Mann" [Lmb. 1993, 368 with false Cu. etymology] ||| WCh.: Mbaaru magà- \& Grnt. magì"person" [Smz. 1978, 29, \#39] || CCh.: Buduma migó "people" [BARTH 1851, 214] = migo "home" [Gaudiche 1938, 20] = mígộu $\sim \operatorname{miggo}(\bar{e})$ "Menschen, Männer" [Nct. apud Lks. 1939], Afade mégu "Menschen" [Stz.], Ngala māgo "people" [Barth/Benton], Klesem mago "people” [Barth] (Ktk.: Slk. 1967, 231-2, §223).

540. AA *m-g- $\boldsymbol{h}$ "to do good" > SBrb.: EWlm. \& Ayr măgg-ăt "faire du bien à, bienfait, bonne nouvelle, grâce, volonté de faire des bienfaits" [PAM 2003, 526] ||| SCu. *magah- "to treat well, behave properly toward" [Ehr.]: Irq. magah- "to take care of" | Qwd. magah-am"to be good" (SCu.: EHRET 1980, 342, \#7). 
541. AA *m-g-d/t-(h??) "Mörser": NEg. $m \underline{d}$ dh h.t $t<* m g d h-t]$ "mortar" (NE, DLE I 260; cf. Wb II 193, 2) = mdd dh.t "Mörser" (GHWb 383) $\rightarrow$ Cpt.: (SB) $\overline{\mathbf{M}} \mathbf{x} \mathbf{\alpha} 2 \overline{\mathbf{T}} \sim \overline{\mathbf{M}} \mathbf{x} \mathbf{\alpha} \overline{\mathbf{2}}$ "Mörser" (KHW 114; CED 101) || NBrb.: Shilh a-maday [met.?] "meule" [Jst. 1914, 121] ||| NAgaw: Bilin magdər "mortar" [Lmb.] || LECu.: Saho mōgód, pl. måwáged "Mörser" [Rn. 1885, 96; 1890, 260] = Saho \& Afar môga d "mortier" [CR] = Afar madagg-e [met. < *magadd - or sim.] "mortar" [Lmb.] (ECu.-Agaw: Lmb. 1993, 354; 1993, 364-365).

NB: Orig. an ancient PAA *ma- prefix nomen instrumenti formation of a root * $g-t+$ or $* g-t-h$ ? Cf. CCh.: Mandara ágdza dzra "pilon"| Musgu gə̣da "piler" (CCh.: Mch. 1950, 44).

542. AA * $\boldsymbol{m}$ - $\boldsymbol{k}$ (var. * $\boldsymbol{m}-\boldsymbol{g}$ ?) "1. to be(come), appear, 2. (tr.) make" > EBrb.: Audjila e-mmóg [-g<*-k?] "divenire, essere, transformarsi" [Prd. 1960, 165] || SBrb.: EWlm. ə-mmək \& Ayr ə-ṃmək "manière d'être etc." [PAM 2003, 533] ||| NOm.: Omt. (sic) mak- "preparare, apprestare", mok- "levarsi (sole)" [Mrn. 1938, 151] = Wolayta \& Gamu \& Dache mokk- "to emerge", Kachama mokk- "to go in front of s'one" (Omt.: LS 1997, 458)| Magi mamk "preparare" [ToselLi 1939, 38] ||| CCh.: Mandara mag-a "1. sich ereignen, 2. machen, tun" [MiRT 1970-71, 67], Malgwa mága “1. machen, tun, 2. sich ereignen” [LöHR 2002, 301].

543. AA * $\boldsymbol{m}$ - $\boldsymbol{k}$ "owl sp. (?)" > NBrb.: Zayan \& Sgugu muka "chouette" [Lbg. 1924, 569] ||| ECh.: Lele mígi "un oiseau: rapace nocturne" [WP 1982, 62]. Accidental similarity?

544. AA *m-k (?) "bull” > SBrb.: EWlm. \& Ayr ă-maka "grand taureau castré" [PAM 2003, 534] ||| SOm.: Ari mēk- \& mayk "buffalo" [Bnd. 1994, 146], Dime meké [Sbr.] = méke [Flm.] "bull" [Bnd. $1996 \mathrm{MS}, 1, \# 22]$.

545. AA * $\boldsymbol{m}-\boldsymbol{k}$ "to be(come) low" > Sem.: Ug. $m k k \mathrm{G}$ "to fall, flag", $m \boldsymbol{k}$ "depressed and disgusting place" [DUL 542], Hbr. mkk qal "to lower, sink", nifal "to come low", hofal "to be(come) immersed" [KB 580] = "zusammensinken" [WUS \#1561], Aram. mkk "niedrig warden" [WUS] ||| LECu.: Oromo (Orma, Wolane) mekō "iron standard supporting cooking pots on the fire" [Strm. 1987, 366; 2001, 55].

546. AA * $\boldsymbol{m}-\boldsymbol{k}$ "to pound (in mortar)" > Sem. *m $\boldsymbol{k}^{\boldsymbol{k}}$ (root ext. - $\varsigma_{-}$) "to squash" [GT]: Hbr. $m^{\Upsilon} k$ qal "1. to squeeze (breast), 2. squash (testicles), 3. thrust into (a spear into the ground)" [KB 612], MHbr. \& JAram. $m^{\uparrow} k$ "to crush" [KB], PBHbr. $m^{\uparrow} k$ "(z)erdrücken" [DALMAN 1922, 245] = "zerdrücken, zerquetschen" [LEVY 1924 III 190] | presumably Ar. maraka "auf dem Boden reiben oder drücken" [GB 445] = "to rub on the ground, struggle" [KB] ||| LECu.: Afar mokko-ta (f) "mortar", maku-tta "pestle" [PH 1985, 162, 170] ||| NOm.: Yemsa muko "to thresh" [Wdk. 1990, 131] = mùk- "to thresh" [Ehret] ||| WCh.: Bole múkkó "wiederkäuen" [Lks. 1971, 137], Pero múgù "to chew" [Frj. 1985, 42] || CCh.: Sao moki (měki) "Mehl” [DuisBurg 1914, 41] || ECh.: WDng. màkè "topound (in mortar)" [FÉDrY in JI 1994 II 269], EDng. máké "piler au mortier" [Dbr.-Mnt. 1973, 192] = "stampfen" [Ebs. 1979, 128; 1987, 94]. Cf. also NOm.: Yemsa mokoy- "mahlen (durch Stampfen)", mokoyò "Mörser" [Lmb.]?

NB: Eventually might be related to WCh.: PAngas *muk "to squeeze, throttle" [GT 2004, 254]: Angas muk "to press, squeeze" [Flk. 1915, 247] = mùk "würgen" ( $\approx$ Hs. šaakewa, so! cf. Hs. šáákè "1. to strangle, 2. fill chock-

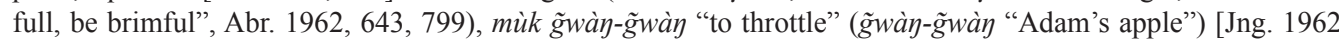
MS, 26], which V.É. Orel (HSED 387, \#1789) falsely equated with Hbr. mkk qal "to lower, sink" [KB] (for its genuine AA etymology cf. above). 


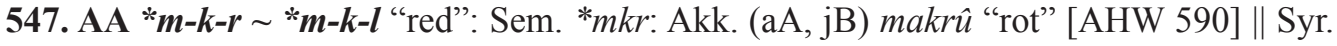
$m k r$ "schwarz sein" [Brk. apud Clc.] || Ar. makira "être rouge", makr-, pl. makūr- "terre rouge avec laquelle on marque en rouge', mumtakir- "marqué de terre rouge" [BK II 1138] = makira "to be red", IX "to be red", makr- "red (noun), red chalk' [Ember] ||| SBrb.: perhaps EWlm. \& Ayr ta-mrrək $\sim t a-m ̧ \partial r \partial k$ "1. marque de propriété faite au fer rouge, 2. fer à marquer, fer rouge" [PAM 1998, 222] ||| CCh.: PMusgu *m-k-l "red” [GT]: Musgu mékele $\underline{\text { (m) }}$, makalai (f) "rot, braun" [KRAUSE apud MüLLER 1886, 400 \& Lks. 1941] = mekelé "rot" [Lks. 1937, 142] = mekele "weiß" [DECORSE apud Lks. 1941, 67] = mękęle "rouge" [Mch. 1950, 37], Kaykay mékélé "rot” [Sgn.-Trn. 1984, 26], Puss mekele (m), makalay (f), pl. makalakay "rouge" [Trn. 1991, 104] | (?) Masa bakāl [irreg. b-<*m-?] "rot" [Lks. 1937, 99].

NB1: V.É. Orel \& O.V. Stolbova (HSED \#1717) combined Sem. *mkr with LEg. $m k r r$ "zwischen schwarz und weiß als Farbenbezeichnung einer Holzart genannt" (GR, Wb II 163, 5), which, however, is not confirmed in PL. NB2: As confirmed to me by K.-G. Prasse (p.c., 6 August 2006), the Tuareg form is not a French loan (not indicated as such in PAM 1.c. either) but rather "it should be a genuine Tuareg word, although it seems to be an isolated word with no other words akin to it of the same root".

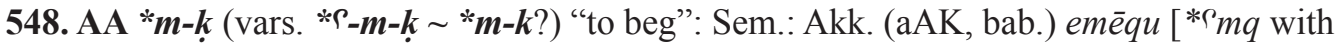

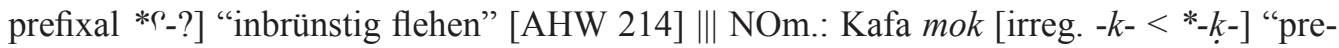
gare, supplicare", mokk-ō "preghiera" [Crl. 1951, 471] || SOm.: Ari míks- (caus. -s-?) "to ask, beg” [Bnd. 1994, 144] ||| WCh.: Bade maak- "suchen" [Lks. 1974-75, 103].

NB1: Since the first syllable of Akk. is not reflected at all in Cu.-Om., I assume that Sem. $* r_{-} / * h$ - was prefix, just like in the preceding AA root (AA * $m-h$ "power").

NB2: E. Cerulli (1.c.) compared Kaffa mok to HECu.: Hadiyya moq-ō, which, however, belongs to a distinct AA root, cf. AA * $m-K$ "to hide" [GT] (below).

549. AA * $\boldsymbol{m}$ - $\boldsymbol{k}$ "little" > NBrb.: Mzg. $\boldsymbol{i}$-miq "peu, un peu (indique surtout la quantité)" [TAIFI 1991, 425] ||| NOm.: Shinasha of Dangur muk-a "small" [Flm. 1990, 28] = mukk-á "klein", mukk-à "wenige", mùk-ǐs- (caus.) "vermindern, verkleinern” [Lmb. 1993, 351-2].

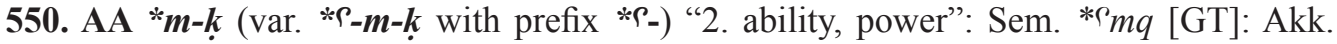
emūqu, nA also amūqu [*ৎmq?] "Armkraft, Macht, Gewalt" [AHW 216] ||| HECu. *mak"power, strength" [GT]: Sidamo maq-é "1. forza, 2. autorità, 3. podestà" [Mrn. 1940, 229] = makaé "strength, power" [Hds.] = maqaè "strength, energy, physical condition, features" [Gsp. 1983, 224], Kmb. mak-o "strength, power" [Hds.] (HECu.: Hds. 1989, 144-5, 384) | Dullay: Tsamay $m u \dot{q}-$ - "to strengthen" [Hyw. 1989, 29] = mù $\dot{q}-u m$ "to be powerful" [SAVÀ 2005 MS, 247] ||| WCh.: AS *mak (var. *muk?) "1. to suffice, be (much) enough, 2. able" [GT 2004, 239-240]: Sura mak "können, vermögen, erreichen, genügen” [Jng. 1963, 73], Mpn. māk "to suffice, satisfy" [Frj. 1991, 35], Kfy. mak "enough", (se) mak àn "enough for me" [Ntg. 1967, 26], Msr. mak $\sim \operatorname{muk}$ (so, -u-) mok (so, -o-) "1. sufficient, 2. plenty, much", mak-čiin "sufficiently, plenty, useful" [Dkl. 1997 MS, 179, 181, 387] = mak" able"

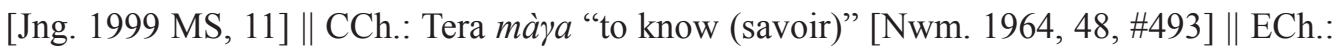
Tumak $m \bar{a} g$ "1. être capable, 2. pouvoir, 3. (être) beaucoup" [Cpr. 1975, 81].

NB1: The first radical of the Sem. root must have been a prefix, as not even the syllable trace of it was preserved in all the other AA reflexes.

NB2: Somali-Jiddu meyg \& Rendille $m \bar{\imath} g$ "Kraft, Macht" do not belong here, deriving ultimately from ECu. *mizg- "right (hand)" [SASSE 1979, 25] (cf. Lmb. 1986, 261). 
551. AA * $\boldsymbol{m}$ - $\boldsymbol{k}$ "to break" > Ar. maqqa "fendre la spathe du palmier femelle pour y introduire la fleur du palmier male" [BK II 1134] ||| NOm. *mek- "to break (intr.)" [Lmb.]: Wolayta me??- [-??- <*-kk-], Gamu \& Dache mekk-, Zayse \& Koyra me?- etc. (NOm.: LS 1997, 449).

552. AA * $\boldsymbol{m}-\boldsymbol{k}$ (var. * $\varsigma_{-} \boldsymbol{m}-\boldsymbol{k}$ with prefix $* \varsigma_{-}$) "long" > Ar. `amiqa "être long, se prolonger, s'étendre loin, être profond", occurs also without ${ }^{\circ}$-, cf. maqaq- "longueur du corps (d'un cheval)" [BK II 369, 1134] ||| WCh.: Hausa múúkéékè "long and thick", mík à "1. to stretch out, 2. (intr.) extend, spread (news), continue one's way, set out", mìká(á) "to become protracted, be stretching os.", mík i “ "weaver's stretching thread in loom for weaving" [Abr. 1962, 682, 673].

NB: This isogloss may preserve the traces of an AA *( ) $-m-k$ " 1 . magnitude, reaching far (or sim.), which was perhaps the carrier of the primary sense of the underlying root."

553. AA * $\boldsymbol{m}$ - $\boldsymbol{k}$ "egg" > HECu.: perhaps Sidamo muk- "to hatch" [Hds. 1989, 77: isolated in HECu.] ||| SOm. *muk- "egg” [GT]: Ari \& Galila \& Hamer \& Karo muk- "egg” [Bnd. 1994, 149, \#24] ||| WCh.: Tangale amak“"egg (rare)” [Jng. 1991, 66; JI 1994 II 122: isolated in Ch.].

554. AA * $\boldsymbol{m}$ - $\boldsymbol{K}$ "part of the face between cheek and jaw": NBrb.: Qbl. a-mayeg, pl. $i$-muyag "un côté du visage, joue et mâchoire” [Dlt. 1982, 528] ||| NAgaw: Xamir mikəa "chin” [BSW 1995, 4] || HECu.: Kmb. mōki (-ta) “cheek” [Hds. 1989, 333] ||| WCh.: Hausa múkààmúkíí múmmúkè "lower jaw", pl. mùkààmùkái "the upper and lower jaws" [Abr. 1962, 682].

NB1: Due to phonological reasons, Kmb. mōki cannot be a reflex of HECu. *bok-o (Kmb. m- $\neq$ HECu. *b$\& \mathrm{Kmb} .-k-\neq$ HECu. $*-k-)$.

NB2: The second PAA radical is not yet fully clear. The three various branches point to three various PAA phonemes: AA *-g (NBrb.) vs. AA *-k (HECu.) vs. *-k (Hausa).

555.AA * $\boldsymbol{m}$ - $\boldsymbol{K}$ "to hide" > HECu.: Sidamo moga "to ambush, be on the look-out, 2. take undue advantage, avail o'self (as a servant who works for sometime to find the occasion to steal)" [Gsp. 1983, 236], cf. Hadiyya moq-ō (translated by Crl.'s informant by Amh. qoytā "colloquio segreto") [Crl. 1951, 471] || WCh.: Hausa mákè “to hide oneself” [Abr. 1962, 645], Gwandara mékye "to hide (intr.)", mákye "to stick (tr.)" [Mts. 1972, 78, 80; Skn. 1977, 26].

556. AA * $\boldsymbol{m}-[\boldsymbol{g}]$ "to calm" > Sem. *mwh: Ug. $m h$ "beruhigt, betrost" [WUS \#1543] = "to prosper" [GORDON 1955, 287, \#1085] |Ar. māha "se calmer (se dit de la colère)" [BK II 1166] = "sich beruhigen" [WUS] ||| NOm.: Haruro māgg-āys "essere content" [CR 1937, 653].

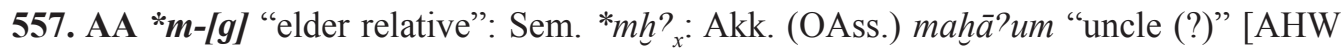
582] = mah $\bar{a}$ ? um or mahh $\bar{a}^{2} u m$ "mng. uncert., seems to indicate a relationship (possibly elder brother or sister) in a family or in a commercial partnership" [CAD m 85] || CCh.: Higi-Kamale muha "old men" [Mkr. 1987, 253] || ECh. *m-K "grandparent" [GT]: Mokilko mògá "grand-mère" \& mùgìyé "grand-père" [Jng. 1990, 140], WDng. mígò (mìgò?) "parent par alliance: beau-père, gendre (terme réciproque)" [FÉDRY 1971, 131], EDng. mìgò "beaupère, gendre" [Dbr.-Mnt. 1973, 204] | Birgit mòkòo-tù "mon grand-père" [Jng. 1973 MS], Toram mogot "grandfather" [AJ 1988 MS, 20].

NB: O.V. Stolbova (1994 MS, 1; HSED \#1801) connected the Akk. term to WCh.: Jimbin muga? a "chief" \| CCh.: Higi dial. maya "chief" | Lgn. myai "chief". Perhaps possible. There can be no connection to Eg. mh.wt "relatives etc." (contra HSED \#1801), since Eg. $\mathrm{h} \neq \mathrm{AA} * \mathrm{Q}$. 
558. AA * $\boldsymbol{m}-\boldsymbol{Q}$ (perhaps * $\boldsymbol{m}-\dot{\boldsymbol{q}}$ ?) "1. sweet, 2. good": Sem.: perhaps Ar. $m q m q$ I "être doux"

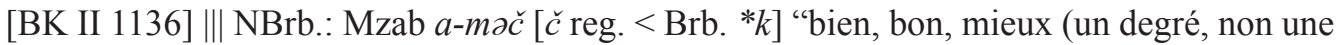
qualité)" [Dlh. 1984, 115] ||| LECu.: Elmolo mak-a "sweet" [Lmb.] = máko? "süß" vs. māk "Honig" [HeIne 1973, 280] = m'áka "sweet" [HeINE 1980, 207], Arbore mákk "sweet thing", makká "sweet", makk-ow- "to taste good" [Hyw. 1984, 383], cf. Somali-Jabarti múki (f), múky-o "Feige" [Rn. 1904, 78] ||| WCh.: Angas myćk myäk (frequently myäk myäk) "tasty, sweet, well flavoured" [Flk. 1915, 251] = myćk [sic with -k] (Ks) "tasty" [Jng. 1962 MS, 27] || CCh.: Bura mimehyu [- $h$ - reg. < AA *-Q-] "sweet and delicious" [BED 1953, 138] | Lamang mak"honey" [Mch. in JI 1994 II 191].

NB: M. LAMBERTI (1993, 362-363; cf. also LS 1997, 450-451) derived the Elmolo cognate mistakenly from OCu. *me - "to be tasty" [Lmb.], which represents a distinct root.

559. AA * $\boldsymbol{m}-\boldsymbol{Q}$ (vars. * $\boldsymbol{m}-\dot{\boldsymbol{q}} \sim *^{*} \boldsymbol{m}-\boldsymbol{g}$ ?) "to carry" > NAgaw *maq $\boldsymbol{w}^{-} / *^{*} \ddot{a} q^{w}-t$ - "to carry on the back" [Apl. $1989 \& 2005]=* m V \gamma^{w}-T$ - "to carry (on the shoulders)" [Apl. 1991] = NAgaw

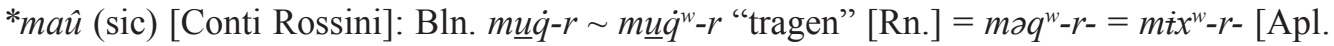

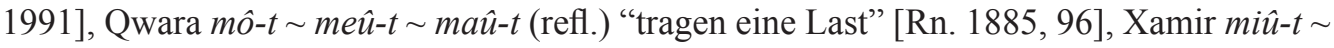
$m \hat{u}-t$ "tragen, sich aufladen" [Rn. 1884, 390], Xamtanga $m \ddot{a} w-t / r-$ [Apl. 1989] = məw-r- "to carry" [Apl. 1991], Qemant mo-t "porter, lever" [CR 1912, 234] = ma $\gamma^{w}-t-\sim m \ddot{a} \gamma^{w}-t-[$ Apl. 1989] $=m \dot{t} \gamma^{w}-t$ - "to carry" [Apl. 1991] (Agaw: Apl. 1989 MS, 16; 1991 MS, 3; 2005 MS, 21) || LECu.: perhaps Rnd. meḥ, pl. meháh [irreg. -h]] "load” [HeIne 1976, 218] | Dullay: Tsamay $m \bar{e} g$ '- "to fetch water" \& Dobase mag'g'- "to ladle up water" [Hyw. 1989, 6, 23] ||| WCh.:

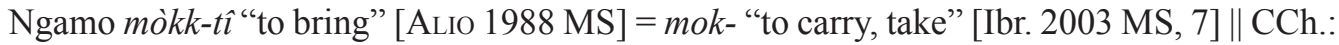
Mulwi $\sqrt{ } m^{w}$-g: mùgí "enlever (une femme), saisir à bras-le-corps" [Trn. 1978, 304] || ECh.: Kera móké "etwas Schweres hochheben" [EBERT 1976, 82] | Bidiya mòg "aider qn. à porter un lourd fardeau" [AJ 1989, 99].

560. AA * $\boldsymbol{m}-\boldsymbol{Q}($ (?) $\sim$ var. * $\boldsymbol{m}-\boldsymbol{h}$ "happy" > Ar. $m \underline{h m} \underline{h}$ [irreg. $-\underline{h}$-] "être gai, joyeux, content" [BK II 1070] ||| HECu.: Burji magg- "to be happy, hope (intr.)", magg-a "happiness" [Hds. 1989, 76] | Dullay: Gollango mokkok- "lächeln" [AMS 1980, 213] || SCu. *măk- "to be happy" [Ehr.]: Alg. maq- "to be cheerful" | Ma'a -máka "to wonder, be astonished" (SCu.: Ehret 1980, 155, \#21). Cf. also Ongota muhi muhi "to laugh" [Flm. 1992, 212].

561. AA * $\boldsymbol{m}-\boldsymbol{Q}-\boldsymbol{r} \sim * \boldsymbol{m}-\boldsymbol{r}-\boldsymbol{Q}$ "old" (?) > Brb. *m- $\boldsymbol{\gamma}-\boldsymbol{r}$ "être grand, vieux, notable" [Dlg.], for which cf., e.g., NBrb.: Wargla $a$-myar "ancien, vieux, notable du clan, de tribu, anciens chefs" [Dlh. 1987, 193], Qbl. u- i-mүur "1. être grand, grandir, 2. être considérable en proportion ou valeur", a-mүar "1. homme âgé, 2. vieillard, 3. beau-parents, (pl.) les anciens, témoins de la tradition" [Dlt. 1982, 508], Irzhen a-myar "vieillard, notable" [Picard], Ait Mgild $a$-myar "old person, leader of performers, head of tribe" [HARRIES 1974, 224] || EBrb.: Gdm. ə-mq $\bar{u} r$ "1. être âgé, ancien, 2. être notable" [LANFrY 1973, 214, \#1019] || SBrb.: Hgr. $a$-myar "homme grand (d'âge, de situation dans sa famille ou dans la société)", $i$-myar "être grand (de dimensions, d'âge, de position sociale)" [Fcd. 1951-2, 235, 237] (Brb.: Bst. 1890, 316; Dlg. 1967, 7, \#3) ||| Ch. *m-r-Q “old” [GT]: CCh.: PLamang *m-l-h [-l-<*-r-poss.] “old” [GT, cf. Büchner 1964, 41-42; WolfF 1971, 65, 69, 71; Mkr. 1987, 279] || ECh.: Bidiya muràk "1. vieillir, 2. s’user”, múrkò “vieux, ancien, âgé” [AJ 1989, 101].

NB1: Root ext. *-r? Any connection with AA * $m-[g]$ "elder relative" (above)?

NB2: Alternatively (provided its primary sense was not "old" but "great"), the Brb. root could be connected with 
Ar. mahara I "5. élargir qqch. à force de se mouvoir dans l'intérieur", ya-mhūur- yu-mhū $r$ - "long, allongé, trop long" [BK II 1072].

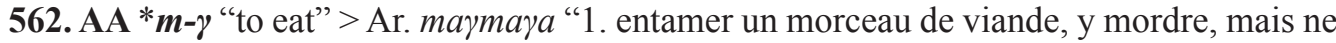
pas l'avaler entièrement, 3 . boire de l'eau avec la langue (se dit des chiens qui boivent ainsi), en fourrant la tête dans un vase" [BK II 1133] ||| NAgaw: Xamir $m \bar{\imath}$ "bread" [Lmb.] (orig. * "food") || LECu.: Saho maw måw "das Mittagsmahl, Mahlzeit" [Rn. 1890, 277] = maw $\sim$ mowo "lunch" [Vergari 2003, 134], Afar $m \bar{a}^{\Upsilon} w$-ó (f) "das Mittagessen, die Hauptmalzeit des Tages" [Rn. 1886, 878] = mayo "dinner, lunch, midday meal, having drunk milk or water, being satisfied with m. or w." [PH 1985, 166] = mā $\bar{c}_{-o}$ "food" (sic) [Lmb.] ||| NOm. *mā "manger" $[\mathrm{Crl}]=.* m$ - "to eat" [Bnd.; Flm. 1983, 453-4] $=*_{m a}$ - "to eat" [LS] $=* m \bar{V}-[\mathrm{GT}]$ (NOm.: Crl. 1937-40, 34; 1938 III, 79, 205; 1951, 466-467; Mkr. 1981, 217-218, \#59.a; Bnd. 1988, 145; LS 1997, 449) || Ch. *m-(y) "to eat" [GT]: WCh.: Waja mịi- "to swallow" [Kwh. 1990, 239] || CCh.: Bata mwà "to eat together", mwàa-tö "eating together, sharing cooked food" [PwEDDON 2000, 58] | Glavda miya "(a kind of) food" [RB 1968, 66] | Lamang may-a "weiden, füttern" [WolfF 1972, 198] || ECh.: Kwang mé "manger (viande)" [Jng. in Brt.-Jng. 1990, 113], Kwang-Mobu mé "manger (viande)" [Jng. in Lenssen 1982, 109; 1984, 69]. From AA *may?- "to eat" [Mlt. 2005, 360, \#23] $=*_{m-y}($ ?) [GT]. Areal parallel: NS *mày or *māy "to chew up" [EHREt 2001, 280, \#110].

563. AA *m- $\uparrow$ "phlegm, slime": Sem. *my "to flow (of efflux?)": Akk. $m a \hat{}$ ? $u$ [irreg. *-a-] (a/jB) "(Galle) erbrechen" [AHW 637] \| Ar. $m y$ `: $m \bar{a} \bar{\Upsilon}^{\circ}$ "1. couler doucement en se répandant à la surface du sol" [BK II 1172] = "to flow (liquid)" [Lsl.] \| Geez my : $m e^{\Upsilon} a$ "1. to become water, melt, liquefy, 2. putrefied" [Lsl. 1987, 376] ||| presumably Eg. mৎৎ (det. of bodily efflux) "etwas vor dem man sich hüten soll” (XVIII. Mag., Wb II 46, 12) = "etwas vor dem man sich hüten muß (in Zshg. mit Lippen)" (GHWb 327) ||| SCu.: Ma'a ma?a-tuko [-?- reg. <*-९-] "phlegm" [Ehret 1980 MS, 4] ||| Ch. *miH- "phlegm” [GT]: WCh.: Gurun-

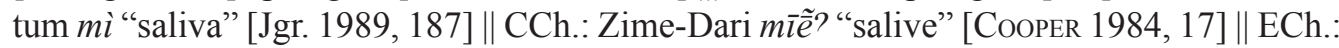
Jegu $m i$ "Rotz" [Jng. 1961, 115].

564. AA *m- $\uparrow$ "side" > Sem.: Sqt. myৎ: mi`eh "côté" [SSL 1991, 1466, cf. Lsl. 1938, 248 with an improbable Sqt. \& Sem. etymology] ||| NBrb.: Senhazha ta-ma, pl. ta-mi-win "pan

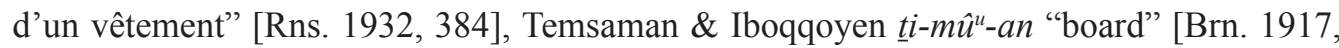
93], Mzab t-ma, pl. ti-mi-wa "côté, direction" [Dlh. 1984, 114], Bugi ta-ma \& Tarudant tsa$m a$ "côté" [Bst. 1890, 312], Qbl. ta-ma, pl. ta-mi-win "1. côté, face, 2. lisière" [Dlt. 1982, 479] || SBrb.: EWlm. \& Ayr tă-ma, pl. tă-ma-wen tă-ma-ten "1. bout, extrémité, 2. limite, périphérie, bord(ure), 3. frontière" [PAM 1998, 207; 2003, 518] ||| LECu.: perhaps Oromo mo'-ō "hip" [GRAGG 1982, 289] ||| CCh.: Gude má "2. edge, rim (from 1. mouth)" [Hsk. 1983, 232].

NB: These forms might well be derived from the root *m "mouth" (as suggested by K.-G. Prasse in PAM 1.c.).

565. AA *m- $\uparrow " 1$. sweet $\rightarrow 2$. good" > Ar. $m \varsigma y: m \bar{a}^{\Upsilon}$ in "doux, mou, tender", cf. $m a^{\Upsilon} w$ "dates" [BK II 1131] || ES: Grg. (< ECu.): End. $m u$ ?, Enm. mo?, Msq. $m^{w} a m^{w} \ddot{a}$ "good, well” (Grg.: Lsl. 1979, 386) ||| LECu. *ma ${ }^{\text {- }}$ [Black] "1. good, 2. sweet” [GT]: Saho ma?_ "3.

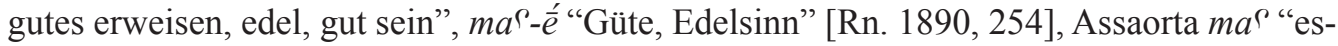
ser buono, prosperare" [CR 1913, 69], Afar ma ${ }^{\uparrow-~ " w e l l, ~ c o n t e n t ", ~ m a ~-o ~ " g o o d " ~[B l a c k] ~}$

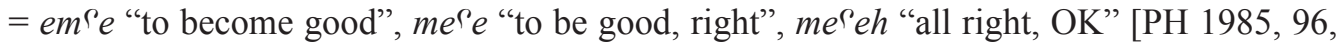


167] | Som. $m a^{\Upsilon}-i$ "Klarheit, Deutlichkeit, Annemlichkeit, Güte, Süßigkeit" [Rn. 1902, 281] $=m a^{\Gamma}$ "esser buono, prosperare" [CR 1913, 69], PBoni *mà̀áà "sweet" [HeInE 1982, 131]: Boni $m a^{7} \bar{a}$ ? "sweet" [Heine] = $m a$ ? $\bar{a}$ [Tosco 1996, 43] | "Macro-Orm." *me - [Black]: Orm. $m i$ ? $a$ - "to taste good" [Black] $=m i$ ' $\bar{a}^{w} a$ "to be expensive, sweet, flavoured" [GRAGG 1982,

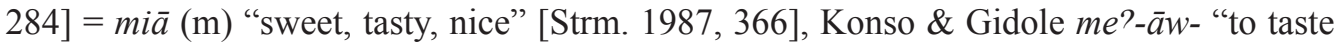
good" [Black] = Konso me?-awa "sweet" [Lmb.] | Dasenech (Galab) me? "sweet" [Black] | HECu.: Sidamo mây(y)e (part.) "alright" [Gsp. 1983, 227] = maw-ō [< *ma? $\bar{c}$ " "good" (ECu.: Rn. 1886, 878; 1902, 281; Crl. 1938 II, 45; Chn. 1947, \#81; Dlg. 1973, 179; SASSE 1973, 268, \#3; Black 1974, 136; Hhn. 1975, 88; Heine 1977, 293; 1978, 69; Blz. 1991, 49) ॥ SCu.: Alg. $m a$ ?-at- $~ m a$ ?-as- [irreg. < *ma - -?] "to be savoury" [EHRET 1980, 323] ||| NOm.: Janjero (Yamma) $m a^{9} \bar{a} \sim m a^{\prime} \bar{o}$ "buono" [Crl. 1938 III, 79] = ma? á "1. nett, 2. Gut" [Lmb. 1993, 362-3] || WCh.: Fyer mó "Süße" [Jng. 1970, 88], Bokkos mimya "sweet" [MAGWA etc. 1985, 10] | Kir mwà- "good" [Smz. 1978, 42, \#91] || CCh.: Muskum míyáwá "bon, beau" [Trn. 1977, 18] || ECh.: Tobanga $m \bar{o} w \bar{a}$ ? "duceur sucrée" [Cpr. 1978, 146] | Sokoro maia "schön, gut" [Lks. 1937, 35].

566. AA * $\boldsymbol{m}-\boldsymbol{h} \sim * \boldsymbol{m}-\boldsymbol{h}$ "to hit" > Ar. $m h w$ : $m a h \bar{a}$ "porter à qqn. un coup violent" [BK II 1164] || SCu. *ma-h̆ "to hit" [Ehr.]: Brg. mah-as- "to beat (person)" | Ma'a -ma "to hit" (SCu.: Ehret 1980, 156, \#28). Irreg. Ar. - $h$ - vs. SCu. *-h- .

567. AA * $\boldsymbol{m}$ - $\boldsymbol{h}$ "bright" > Ar. mahah- mahāh- "éclat, beauté, fraîcheur" [BK II 1159] || Bed. mah $\sim$ meh "morgen werden, tagen, anbrechen der Tag", mah $\sim$ máha "der Morgen, Sonnenaufgang" [Rn. 1895, 164] || LECu.: Saho \& Afar māh "licht werden, Morgen

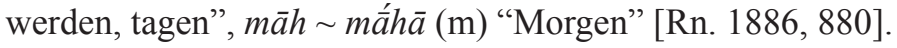

\section{ABBREVIATION OF LANGUAGE NAMES AND RELATED TERMS}

(A): Ahmimic, AA: Afro-Asiatic (Afrasian, Semito-Hamitic), aAk: Old Akkadian, aAss.: Old Assyrian, aB: Old Babylonian, Akk.: Akkadian, Alg.: Alagwa, Amh.: Amhara, -ic, Ar.: Arabic, Aram.: Aramaic, AS: Angas-Sura, Ass.: Assyrian, (B) Bohairic, Bab.: Babylonian, BA(ram.): Biblical Aramaic, Bch.: Bauchi, Bed.: Bed'awye (Beja), BM: Bura-Margi, BN: Bade-Ngizim, Brb.: Berber (Libyo-Guanche), Brg.: Burunge, BT: Bole-Tangale, C: Central, Ch.: Chadic, Cpt.: Coptic, CT: Coffin Texts, Cu.: Cushitic, Dem.: Demotic, Dhl.: Dahalo, E: East, Ebl.: Eblaite, Eg.: Egyptian, ES: Ethio-Semitic, ESA: Epigraphic South Arabian, Eth.: Ethiopian, Eth.-Sem.: Ethio-Semitic, (F): Fayyumic, Gdm.: Ghadames, Gmy.: Goemay, GR: Ptolemaic and Roman period, Grg.: Gurage, Grw.: Gorowa, H: Highland (in Cushitic), Hbr.: Hebrew, Hgr.: Ahaggar, Hrs.: Harsusi (in MSA), IE: Indo-European, Irq.: Iraqw, irreg.: irregular, Izn.: Iznasen, JA(ram.): Jewish or Judeo-Aramaic, jB: jungbabylonisch, Jbl.: Jibbali, Kfy.: Kofyar, KK: Kera-Kwang group, L: Late, L: Low(land), lit.: literature, Lit.: literary texts, LL: lexical lists, LP: Late Period, M: Middle, Mag.: magical texts, mA: Middle Assyrian, mB: Middle Babylonian, Math.: mathematical papyri, Med.: medical texts, MK: Middle Kingdom, MM: Mafa-Mada group, Mnt.: Montol, Mpn.: Mupun, MSA: Modern South Arabian, Msr.: Mushere, Mzg.: Tamazight (Beraber), N: New, N: North, nA: New Assyrian, nB: New Babylonian, NC: Niger-Congo, NE(g.): New Egyptian, Nil.: Nilotic, NK: New Kingdom, NS: Nilo-Saharan, Nslm.: Taneslemt, O: Old, OInd.: Old Indic (Sanskrit), OK: Old Kingdom, Om.: Omotic, OSA: Old South Arabian, OT: Old Testament, P: Proto-, PB: Post-Biblical, PT: Pyramid Texts, Qwd.: Qwadza, reg.: regular, S: South, (S): Sahidic, Sab.: Sabaean, Sem.: Semitic, spB: Late Babylonian, Sqt.: Soqotri, Syr.: Syriac, TA(ram).: Aramaic of Talmud, Tna.: Tigrinya, Ug.: Ugaritic, W: West, Wlm.: Tawllemmed. 


\section{ABBREVIATIONS OF AUTHOR NAMES}

Abr:: Abraham, AF: Adolf Friedrich, AJ: Alio \& Jungraithmayr, Ajl:: Ajello, AlC: Angas Language Committee, Alm.: Alema-Yehu, AMS: Amborn \& Minker \& Sasse, Apl.: Appleyard, Aplg.: Applegate, Ast.: Aistleitner, Bgn.: Beguinot, BK: Bieberstein \& Kazimirski, Blv.: Belova, Blz.: BlažeK, Bnd.: Bender, Brk.: Brockelmann, Brn.: Brunet, Brt.: Barreteau, Bst.: Basset, BSW: Berhanu \& Sisay \& Wedekind, Chn.: Cohen, Clm.: Colombel, Cpr.: Caprile, CR: Conti Rossini, Crl.: Cerulli, Crn.: Carnochan, Csp.: Cosper, Ctc.: Caïtucoli, Dbr.: Duibrine, Dkl.: Diyakal, Dlg.: Dolgopolsky, Dlh.: Delheure, Dlt.: Dallet, Ebs.: Ebobisse, EEN: Ehret \& ElderKin \& Nurse, Egc.: Eguchi, Ehr.: Ehret, Fcd.: Foucauld, Flk.: Foulkes, Flm.: Fleming, FMlr.: F. Müller, Frj.: Frajzyngier, Frz.: Fronzaroli, GB: Gesenius \& Buhl, Gcl.: Gochal, Grb.: Greenberg, Grt.: Grottanelli, Gsp.: Gasparini, GT: Takács, Hds.: Hudson, Hfm.: Hoffmann, Hlw.: Hellwig, Hnrg.: Huehnergard, Hsk.: Hoskison, Hyw.: Hayward, Ibr.: Ibriszimow, IL: Institute of Linguistics, IS: Illič-Svityč, JA: JungraithmaYr \& Adams, Jgr: JagGar, JI: Jungraithmayr \& Ibriszimow, Jng.: Jungraithmayr, Jns.: Johnstone, JS: Jungraithmayr \& Shimizu, Jst.: Justinard, KB: Koehler \& Baumgartner, Krf.: Kraft, Ksm.: Kossmann, Kwh.: Kleinewillinghöfer, Lbf.: Lebeuf, Lbg.: Loubignac, LH: LitTMAnN \& Höfner, Lks.: Lukas, Lmb.: Lamberti, Lnf.: Lanfry, Lns.: Lenssen, LS: Lamberti \& Sottile, Lsl.: Leslau, Lst.: Laoust, Mch.: Mouchet, Mgw.: Maghway, Mkr.: Mukarovsky, Mnh.: Meinhof, Mlt.: Militarev, Mnt.: Montgolfier, MQK: Mous \& Qorro \& Kiessling, Mrc.: Mercier, Mrn.: Moreno, MSkn.: M. Skinner, Mts.: Matsushita, Ncl.: Nicolas, Nct.: Nachtigal, Nhl.: Nehlil, Ntg.: Netting, Nwm.: Newman, NZ: Naït-Zerrad, OS: Orel \& Stolbova, PAM: Prasse \& Alojaly \& Mohamed, PH: Parker \& Hayward, Pls.: Pilszczikowa, Pnc.: Penchoen, Prd.: Paradisi, Prs.: Prasse, Pwd.: Pweddon, RB: Rapp \& Benzig, RK: Reutt \& Kogan, Rn.: Reinisch, Rns.: Renisio, Rpr.: Roper, Rsg.: Rossing, Sbr.: Siebert, Scn.: Sachnine, Slk.: Sölken, Skn.: N. Skinner, Smz.: Shimizu, Srl.: Sirlinger, Ss.: Sasse, SS: Simeone-Senelde, SS: Simeone-Senelle \& Lonnet, Stl.: Stolbova, Str.: Strümpell, Strm.: Stroomer, Sts.: Starostin, TB: Tucker \& Bryan, Tf.: Taïfi, Trn.: Tourneux, TSL: Tourneux \& Seignobos \& Lafarge, Vcl.: Vycichl, Vrg.: Vergote, Wdk.: Wedekind, WP: Weibegué \& Palayer, Wtl.: Whiteley, Zbr.: Zaborski, Zhl.: Zyhlarz.

\section{REFERENCES}

ABÈs Mohamed. 1916 (or shortly after). Manuel de berbère marocain. Place and publisher not indicated. Abraham R.C. 1962. Dictionary of the Hausa Language. ${ }^{2}$ London: University of London Press. Abraham R.C. 1964. Somali-English Dictionary. ${ }^{2}$ London: University of London Press. AEO I-II = GARdInER Alan Henderson. 1947. Ancient Egyptian Onomastica. I-II. Oxford: Clarendon Press. AEPT $=$ FAuLKNER Raymond Oliver. 1969. The Ancient Egyptian Pyramid Texts. I. Oxford: Clarendon Press. AHW $=$ SodEN Wolfram von. 1965-1981. Akkadisches Handwörterbuch. I-III. Wiesbaden: Otto Harrassowitz. AistleitNer Josef. 1948. "Untersuchungen zum Mitlautbestand des Ugaritisch-Semitischen.” In: Löwinger \& Somogyi 1948: 209-225.

Ajello Roberto, Karyo Mayore, Melis Antonio, Dobio Ousmanou. 2001. Lexique comparatif de six langues tchadique central (Gizey, Ham, Lew, Marba, Masa, Musey). Pisa: Edizioni Plus, Università di Pisa.

AL I = Meers Dimitri. 1977. Année lexicographique. Égypte ancienne. Tome 1. 2ème edition 1998. Paris: Cybele.

AL II = Meers Dimitri. 1978. Année lexicographique. Égypte ancienne. Tome 2. 2ème edition 1998. Paris: Cybele.

AL III = Meers Dimitri. 1979. Année lexicographique. Égypte ancienne. Tome 3. 2ème edition 1998. Paris: Cybele.

ALC 1978 = Angas Language Committee (in Cooperation with Nigeria Bible Translation Trust): Shàk nkary kè shəktok mwa ndon Ngas. Ngas-Hausa-English Dictionary with Appendix Showing Some Features of Ngas Grammar. Jos, Nigeria: Nigeria Bible Translation Trust.

Alemayehu Abebe. 1993. "Ometo Dialect Survey - A Pilot Survey Report.” Survey of Little-Known Languages of Ethiopia (S.L.L.E.) Reports 4, 1-10.

Auıo Khalil. 1988. Wordlist of Galembi. MS. Frankfurt a/M, 28 August 1988.

Alıo Khalil. 1988. Wordlist of Maha. MS. Frankfurt a/M, 28 August 1988.

Alıo Khalil. 2004. "Préliminaires à une étude de la langue Kajakse d'Am-Dam, de Toram du Salamat, d'Ubi du Guéra et de Masmaje du Batha-Est (Tchad).” In: TAKÁcs 2004b: 229-285. 
Alio Khalil, JungraithmaYr Herrmann. 1988. Allgemeine Kulturwortliste vom Toram. MS. Frankfurt a/M, 28 August 1988.

Alio Khalil, Jungraithmayr Herrmann. 1989. Lexique bidiya. Frankfurt am Main: Vittorio Klostermann.

AllatI Abdelaziz. 1986. Phonétique et phonologie d'un parler amazigh du Nord-Est marocain (le parler des Aït Saïd). Tome III. Thèse de doctorat de 3ème cycle. Université de Provence, Centre d'Aix-en-Provence.

AMS = Amborn Hermann, Minker Günter, Sasse Hans-Jürgen. 1980. Das Dullay. Materialen zu einer ostkuschitischen Sprachgruppe. Berlin: Reimer Verlag.

Appleyard David. 1989. Agaw and Omotic Links. The Evidence of the Lexicon. MS. Paper presented at the 2nd International Symposium on Cushitic and Omotic Languages, Torino, November 1989. Proceedings forthcoming.

APPLEYARD David. 1989. Agaw Vocabulary Comparative Notes. MS. London.

ApPLEYARD David. 1991. A Comparative Agaw Wordlist. MS. London.

Appleyard David. 2005. Comparative Agaw Dictionary. MS. Printout of 3 Feb. 2005.

BARRETEAU Daniel. 1995. "Vowel and Tonal Variations within the Consonantal Framework of the Verbal System in Central Chadic Languages." In: IBRISZIMOw \& Leger 1995: 197-228.

Barreteau Daniel, Blérs Yvonne. 1990. Lexique mafa. Langue de la famille tchadique parlée au Cameroun. Paris: ORSTOM, Librairie Orientaliste Paul Geuthner.

Barreteau Daniel, JungraithmaYr Herrmann. "Les verbes monoradicaux dans les langues tchadiques." In: JunGRAITHMAYR \& TOURNEUX 1990: 37-214.

BARTH Henry. 1851. "Vocabulary of Budduma, Spoken by the Inhabitants of the Islands in Lake Chad." Journal of the Royal Geographical Society 21, 214.

BASSET René. 1885. "Notes de lexicographie berbère." Journal Asiatique, Ser. VIII, 5, 148-198.

BASSET René. 1890. Le dialecte de Syouah. Paris: Ernest Leroux.

BASSET René. 1890. Loqmân berbère avec quatre glossaires et une étude sur la légende de Loqmân. Paris: Ernest Leroux.

BASSET René. 1909. Mission au Sénégal. Tome I. Étude sur le dialecte zenaga. Paris: Ernest Leroux.

Bechraus-Gerst Marianne, Serzisko Fritz (eds.). 1988. Cushitic-Omotic. Papers from the First International Symposium on Cushitic and Omotic Languages, Cologne, January 6-9, 1986. Hamburg. Helmut Buske Verlag.

BED = Anonymous. 1953. Bura-English Dictionary. (Place unknown, publisher unnamed). Master copy in the library of the Seminar für Afrikanische Sprachen und Kulturen der Universität Hamburg (inv. no.: 15748 / JT 1526).

Beguinot Francesco. 1931. Il berbero Nefûsi di Fassâțo. Roma: Istituto per l'Oriente.

Beguinot Francesco. 1942. Il berbero Nefûsi di Fassâtọ. Roma: Istituto per l'Oriente.

Behnstedt Peter. 1987. Die Dialekte der Gegend von S'ådah (Nord-Jemen). Wiesbaden: Harrassowitz.

Belova Anna. 1987. "Struktura kornja v drevneegipetskom i semitskih jazykah." In: Problemy języków Azji i Afryki. Warszawa: Wydawnictwo Polskiej Akademii Nauk, 275-282.

Belova Anna. 1988. "The Position of Himyaritic within the South Semitic Group of Semitic Languages (Yemenite-Ethiopian Isoglosses)." In: GroмYко 1988: 28-34.

Belova Anna. 1996. "Einige jemenitisch-afrikanische Isoglossen.” In: Griefenow-Mewis \& Voigt 1996: 33-42.

BENDER Marwin Lionel. 1974. Wordlist of Chara. MS. Carbondale.

Bender Marwin Lionel. 1988. "Proto-Omotic Phonology and Lexicon.” In: Bechraus-Gerst \& Serzisko 1988: 121-159.

Bender Marwin Lionel. 1990. “The Limits of Omotic.” In: HaYward 1990: 584-616.

Bender Marwin Lionel. 1994. "Aroid (South Omotic) Lexicon.” Afrikanistische Arbeitspapiere 38, 133-162.

BENDER Marwin Lionel. 1994. "The Mystery Languages of Ethiopia." In: Marcus 1994: 1153-1174.

Bender Marwin Lionel. 1996. "The Limits of Omotic Revisited.” In: Griefenow-Mewis \& Voigt 1996: 143-166.

Bender Marwin Lionel. 2003. Omotic Lexicon and Phonology. Carbondale: Southern Illinois University.

Berhanu Hailu, Sisay Dereje, Wedekind Klaus. 1995. "Khamir - the People ['ximra] and Their Language [xim'tana]." Survey of Little-Known Languages of Ethiopia Linguistic Reports 23, 1-8.

Biarnay S. 1911. Études sur le dialecte des bet't'ioua du Vieil-Arzen. Paris: Typographie Adolphe Jourdan (= Revue Africaine 277, 1910, 1-261).

Biarnay S. 1917. Étude sur les dialectes berbères du Rif. Lexique, textes et notes de phonétique. Paris: Ernest Leroux.

Bieberstein Kazimirski A. de. 1860. Dictionnaire arabe-français. I-II. Paris: Maisonneuve et C ${ }^{\text {ie }}$. 
Black Paul David. 1974. Lowland East Cushitic: Subgrouping and Reconstruction. Ph.D. dissertation. Yale University.

BlažeK Václav. 1991. "Honey in Cushitic and Omotic Languages." Afrikanistische Arbeitspapiere 26, 37-66.

Bouny Paul. 1975. Wordlist of Kotoko. MS. N'Djamena.

Boyd Raymond. 2002. Bata Phonology. München: Lincom Europa.

Brockelmann Carl. 1932. “Ägyptisch-semitische Etymologien.” Zeitschrift für Semitistik 8, 97-117.

BüChNer H. 1964. "Vokabulare des Sprachen in und um Gava (Nordnigerien)." Afrika und Übersee 48, 36-45.

Bynon J. (ed.). 1984. Current Progress in Afro-Asiatic Linguistics. Amsterdam: John Benjamins.

BYNon J., Bynon Th. (eds.). 1975. Hamito-Semitica. The Hague: Mouton.

$\mathrm{CAD}=$ The Assyrian Dictionary of the Oriental Institute of the University of Chicago. Vol. 1-21. Glückstadt-Chicago, Since 1956, J. J. Augustin, The Oriental Institute of the University of Chicago.

CAḯtucoli Claude. 1983. Lexique masa. Paris: Agence de Coopération Culturelle et Technique.

CAÏTUCOLI Claude. 1978. "Schèmes tonals et morphologie du verbe en masa." In: CAPRILE \& JungraithmaYr 1978: 67-87.

CAPRILE Jean-Pierre. 1972. Kebe mo:nde (gabri). MS. Djoun: Commune de Laï, février.

CAPRILE Jean-Pierre. 1975. Lexique tumak-français (Tchad). Berlin: Verlag von Dietrich Reimer.

CAPRILE Jean-Pierre (ed.). 1977. Études phonologiques tchadiennes. Paris: Socété d'Études Linguistiques et Anthropologiques de France.

CAPRILE Jean-Pierre. 1978. "Les mots voyageurs dans l'interfluve Bahr-Erguig/Chari/Logone: comparaison lexicale entre le barma, langue sara de l'ancien royaume baguirmien, et le tumak, langue tchadique." In: CAPRILE \& JUNGRAITHMAYR 1978: 145-156.

CAPRILE Jean-Pierre. 1978. "Notes linguistiques sur le tobanga à partir d'un conte en cette langue.” In: JungRAITHMAYR \& CAPRILE 1978: 121-175.

CAPrile Jean-Pierre, JungratthmaYr Herrmann (eds.). 1978. Préalables à la reconstruction du proto-tchadique. Paris: SELAF.

CARnochan Jack. 1975. "Bachama and Chadic.” In: Bynon \& Bynon 1975: 459-468.

$\mathrm{CD}=$ Crum Walter Ewing. 1939. A Coptic Dictionary. Oxford: Oxford University Press.

CED = ČERnÝ Jaroslav. 1976. Coptic Etymological Dictionary. London-Cambridge: Cambridge University Press.

CERulli Enrico. 1929. "Note su alcune popolazioni sidāmā dell'Abissinia meridionale II: i Sidama dell'Omo." Rivista degli Studi Orientali 12, 1-69.

Cerulli Enrico. 1938. Studi etiopici. II. La lingua e la storia dei Sidamo. Roma: Istituto per l'Oriente.

Cerulli Enrico. 1937-1940. "La racine monosyllabique (consonne + voyelle ou voyelle seule) en couchitique." GLECS 3, 33-36.

Cerulli Enrico. 1938. Studi etiopici. III. Il linguaggio dei Giangerò ed alcune lingue Sidama dell'Omo (Basketo, Ciara, Zaissè). Roma: Istituto per l'Oriente.

CERULli Enrico. 1951. Studi etiopici. IV. La lingua caffina. Roma: Istituto per 1'Oriente.

ChaKer Salem, ZABOrSki Andrzej (eds.). 2000. Études berbères et chamito-sémitiques. Mélanges offerts à KarlG. Prasse pour son $70^{e}$ anniversaire. Paris-Louvain: Éditions Peeters.

CoHEn David (ed.). 1970. Mélanges Marcel Cohen. Paris: Mouton.

CoHen Marcel. 1947. Essai comparatif sur le vocabulaire et la phonétique du chamito-sémitique. Paris: Librairie Ancienne Honore Champion.

Colombel Véronique de. 1986. Phonologie quantitative et synthématique. Propositions méthodologiques et théoriques avec application à l'ouldémé (langue tchadique du Nord-Cameroun). Paris: SELAF.

Colombel Véronique de. 1997. La langue ouldémé (Nord-Cameroun). Précis de grammaire, texte, lexique. Paris: Association de Linguistique Africaine.

ConTi Rossin Carlo. 1905. "Note sugli agau. 1. Appunti sulla lingua khamta dell'Averghellé." Giornale della Società Asiatica Italiana 17(2), 183-242.

Conti Rossini Carlo. 1905. "Note sugli agau. 2. Appunti sulla lingua Awiyā del Danghelà." Giornale della Società Asiatica Italiana 18, 103-194.

Contr Rossini Carlo. 1912. La langue des Kemant en Abyssinie. Wien: Alfred Hölder.

Conti Rossini Carlo. 1913. Schizzo del dialetto saho dell'alta Assaorta in Eritrea. Roma: Tipografia della R. Accademia dei Lincei.

Conti Rossini Carlo. 1913. "Studi su populazioni dell'Etiopia." Rivista degli Studi Orientali 6, 365-426.

ConTi Rossini Carlo. 1925. "Sui linguaggi dei Naa e dei Ghimirra (Sce) nell'Etiopia Meridionale." Rendiconti della Reale Accademia dei Lincei, Classe di Scienze morali, storiche e filologiche, ser. VI, vol. 1, 512-636. 
ConTI Rossini Carlo. 1937. "Contributi per la conoscenza della lingua Haruro (Isole del Lago Margherita).” Rendiconti della Reale Accademia Nazionale dei Lincei, classe di scienze morali, storiche e filologiche, ser. VI, vol. XII, fasc. 7-10, 621-679.

CoOPER Koye Nadji. 1984. Lexique zime-français. Vūn tàrí. Sarh (Tchad): Centre d'Études Linguistiques.

Cosper Ronald. 1994. South Bauchi Lexicon. A Wordlist of Nine South Bauchi (Chadic) Languages and Dialects. Halifax: The Author (Saint Mary's University).

DaKubu M.E. Kropp (ed.). 1976. West African Language Data Sheets. Vol. I. (Place not indicated): West African Linguistic Society.

Dallet J.-M. 1982. Dictionnaire qabyle-français. Parler des At Mangellat (Algerie). Paris: SELAF (Société d'études linguistiques et anthropologiques de France).

Dalman Gustaf H. 1922. Aramäisch-neuhebräisches Handwörterbuch zu Targum, Talmud und Midrasch. Frankfurt a/M: J. Kaufmann Verlag.

DCT = Molen Rami van der. 2000. A Hieroglyphic Dictionary of Egyptian Coffin Texts. Leiden: E.J. Brill.

Delheure Jean. 1984. Dictionnaire mozabite-français. Paris: Société d'Études Linguistiques et Anthropologique de France (SELAF).

Delheure Jean. 1987. Dictionnaire ouargli-français. Paris: Société d'Études Linguistiques et Anthropologique de France (SELAF).

DG = ERICHSEN Wolja. 1954. Demotisches Glossar. Koppenhagen: Ejnar Munksgaard.

Diakonoff Igor M. 1965. Semito-Hamitic Languages. Moscow: Nauka.

DiaKonOFF Igor. 1981. "Earliest Semites in Asia. Agriculture and Animal Husbandry According to Linguistic Data (VIIIth-IVth Millennia B.C.)." Altorientalische Forschungen 8, 23-74.

DiakonofF Igor. 1984. "Letter to the Conference." In: Bynon 1984: 1-10.

DiAKONOFF Igor. 1988. Afrasian Languages. Moscow: Nauka.

DiaKonOFF Igor. 1992. "Proto-Afrasian and Old Akkadian." Journal of Afroasiatic Languages 4(1): 1-133.

D'Jakonov Igor', Militarev Aleksandr, Porhomovskij Viktor, Stolbova Ol'ga. 1987. “Obščeafrazijskaja fonologičeskaja sistema.” In: PoRHOMOvsKiJ 1987: 9-29.

Diakonoff Igor, Militarev Alexander, Porkhomovsky Victor, Stolbova Olga. 1993. "On the Principles of Afrasian Phonological Reconstruction.” St. Petersburg Journal of African Studies 1, 7-15.

DiYAKal Philibus. 1997. Mushere-English Dictionary. Collection of words carried out by Mr. Ph. I. D. started on September 10th, 1997 under the supervision of Herrmann Jungraithmayr (Univ. of Frankfurt). MS.

Disirine Bada Adoum Zaid, Montgolfier Paul de (et beaucoup d'autres...). around 1973. Vocabulaire dangaléat. Kawo dayla. Place not indicated, publisher not indicated.

DLE = Lesko Leonard H. A Dictionary of Late Egyptian. Vol. I (1982), II (1984), III (1987), IV (1989). Berkeley: B.C. Scribe Publications.

Dolgopol'skiJ Aron. 1966. “Materialy po sravnitel'no-istoričeskoj fonetike kušitskih jazykov. Gubnye i dental'nye smyčnye v načal'nom položenii." In: UsPENSKIJ 1966: 35-88.

DolgopolsKi Aharon. 1967. "La permutation des * $m$ et * $b$ initiaux dans les racines couchitiques." In: Il Congrès International des Africanistes. Communications de la délégation de l'URSS. Moscou: Nauka, 3-17.

DolgopolsKi Aharon. 1970. "A Long-Range Comparison of Some Languages of Northern Eurasia. Problems of Phonetic Correspondences." In: VII Meždunarodnyj kongress antropologičeskih i étnografičeskih nauk. Moskva, 3-10 avgusta $1964 \mathrm{~g}$. Vol. V. Moskva: Nauka, 620-628.

Dolgopol'skis Aron. 1973. Sravnitel'no-istoričeskaja fonetika kušitskih jazykov. Moskva: Nauka.

Dolgopolsky Aharon. 1983. "Semitic and East Cushitic. Sound Correspondences and Cognate Sets." In: SegERT \& Bodrogligeti 1983: 123-142.

Dolgopolsky Aharon. 1992. From Proto-Semitic to Hebrew: Phonology. Etymological Approach in a HamitoSemitic Perspective. MS. Haifa.

DolgopolsKy Aharon. 2005. "Emphatic and Plain Voiceless Consonants in Hamito-Semitic in the Light of Internal and External Comparative Evidence.” In: FronZaroli \& Marrassini 2005: 29-34.

Duisburg A. von. 1914. "Überreste der Sso-Sprache.” Mitteilungen des Seminars für Orientalische Sprachen 17, $39-45$.

DUL = Olmo Lete Gregorio del, SANMARTín Joaqín. 2003. A Dictionary of the Ugaritic Language in the Alphabetic Tradition. Part One [ $\mathrm{g}(\mathrm{a} / \mathrm{i} / \mathrm{u})-\mathrm{k}]$. Part Two [1-z]. Leiden: E.J. Brill.

Eвert Karen H. 1976. Sprache und Tradition der Kera (Tschad). Vol. II. Berlin: Dietrich Reimer.

EвoBisse Carl. 1979. Die Morphologie des Verbs im Ost-Dangaleat (Guera, Tschad). Berlin: Dietrich Reimer Verlag. 
EвоBisse Carl. 1987. Les verbaux du dangaléat de l'est (Guera, Tchad). Lexiques français-dangaléat et allemanddangaléat. Berlin: Dietrich Reimer Verlag.

EDE I = TAKÁCS Gábor. 1999. Etymological Dictionary of Egyptian. Vol. 1: A Phonological Introduction. Leiden: E.J. Brill.

EDE II = TAKÁcs Gábor. 2001. Etymological Dictionary of Egyptian. Vol. 2: $b$-, p-, f-. Leiden: E.J. Brill.

Edwards I.E.S. (ed.). Hieratic Papyri in the British Museum. Fourth Series. Oracular Amuletic Decrees of the Late New Kingdom. I-II. London 1963.

EG 1927 = GARDINER Alan Henderson. 1927. Egyptian Grammar. ${ }^{1}$ Oxford: Clarendon Press.

Eguch Paul Kazuhisa. 1971. "Matériaux pour servir à l'étude de la langue hidé. Vocabulaire." Kyoto University African Studies 6, 195-283.

EHRET Christopher. 1974. Ethiopians and East Africans. The Problem of Contacts. Nairobi: East African Publishing House.

EHRET Christopher. 1980. The Historical Reconstruction of Southern Cushitic Phonology and Vocabulary. Berlin: Dietrich Reimer Verlag.

EHret Christopher. 1980. Kw'adza Vocabulary. MS. Los Angeles, California.

EHREт Christopher. 1987. "Proto-Cushitic Reconstruction." Sprache und Geschichte in Afrika 8.

EнREт Christopher. 1991. "The Consonant Inventory of Proto-Eastern Cushitic." Studies in African Linguistics 22(3), 211-275.

EHret Christopher. 1995. Reconstructing Proto-Afroasiatic (Proto-Afrasian). Vowels, Tone, Consonants, and Vocabulary. Berkeley-Los Angeles, California: University of California.

EHRET Christopher. 1997. (Additions to the Afroasiatic Reconstructions.) MS. Los Angeles, California.

EHRET Christopher. 2001. A Historical-Comparative Reconstruction of Nilo-Saharan. Köln: Köppe Verlag.

Ehret Christopher, Ali Mohamed Nuuh. 1984. “Soomaali Classification.” In: Labahn 1984: 201-269.

Ehret Christopher, Posnansky M. (eds.). 1982. The Archaeological and Linguistic Reconstruction of African History. Berkeley: University of California Press.

Ehret Christopher, Elderkin E.D., Nurse Derek. 1989. "Dahalo Lexis and Its Sources." Afrikanistische Arbeitspapiere 18, 5-49.

EMBer Aaron. 1914. “Several Semito-Egyptian Particles.” Zeitschrift für Assyriologie 28(2-4), 302-306.

EnnUlat Jürgen. 1976. “Fali.” In: Dakubu 1976.

FaulKner Raymon Oliver. 1969. The Ancient Egyptian Pyramid Texts. I. Oxford: Clarendon Press.

FaulKner Raymon Oliver. 1973. The Ancient Egyptian Coffin Texts. I. Warminster: Aris and Phillips.

FD = FAUlKNER Raymon Oliver. 1962. A Concise Dictionary of Middle Egyptian. Oxford: Clarendon Press.

FÉDRY Jacques (avec la collaboration de KhAmis Jonas \& o/NedJei Moussa). 1971. Dictionnaire dangaleat (Tchad). Thése de 3ème cycle, Institut National des Langues et Civilisations Orientales. Lyon: Afrique et Langage.

FÉDRY Jacques. 1977. "Aperçu sur la phonologie et la tonologie de quatre langues de groupe «mubi-karbo» (Guera) (dangaléat-est, dangaléat-ouest, bidiyo, dyongor).” In: CAPRILE 1977: 87-112.

Fleming Harold C. 1983. "Chadic External Relations.” In: Wolff \& Meyer-Bahlburg 1983: 17-31.

Fleming Harold C. 1990. "Omotica, Afrasiana and More: Ethiopia as the Ever-Flowing Vase." Mother Tongue $12,22-30$.

Fleming Harold C. (jointly with Aklilu Yilma, Ayyelew Mitiku, Hayward Richard, Miyawaki Yukio, Mikesh Pavel \& Seelig J. Michael). 1992. "Ongota (or) Birale: A Moribund Language of Gemu-Gofa (Ethiopia)." Journal of Afro-Asiatic Languages 3(3), 181-225.

Foucauld Charles de. 1951-1952. Dictionnaire touareg-français, dialecte de l'Ahaggar. Vol. I-IV. Paris: Imprimerie Nationale de France.

Foulkes H.D. 1915. Angass Manual. Grammar, Vocabulary. London: Kegan Paul, Trench, Trübner and Co.

Frajzyngier Zygmund. 1985. A Pero-English and English-Pero Vocabulary. Berlin: Dietrich Reimer Verlag.

FrajZyngier Zygmund. 1991. A Dictionary of Mupun. Berlin: Dietrich Reimer Verlag.

Frick Esther J. 1976. "Dghwede.” In: DaKUBU 1976.

Fronzaroli Pelio, Marrassini Paolo (eds.). 2005. Proceedings of the 10th Meeting of Hamito-Semitic (Afroasiatic) Linguistics (Florence, 18-20 April 2001). Quaderni di Semitistica 25. Firenze: Dipartimento di Linguistica, Università di Firenze.

GASPARINI Armido. 1983. Sidamo-English Dictionary. Bologna: E.M.I.

GaUdiche (?). 1938. "Lalangue boudouma.” Journal de la Société des Africanistes 8, 13-32. 
GB = GesenIus Wilhelm (bearbeitet von BuHL Frants). 1962. Hebräisches und aramäisches Handwörterbuch über das Alte Testment. Unveränderter Neudruck der 1915 erschienenen 17. Auflage. Berlin-GöttingenHeidelberg: Springer-Verlag.

GHWb = HANNIG Rainer. 1995. Grosses Handwörterbuch Ägyptisch-Deutsch (2800-950 v. Chr.). Mainz: Verlag Philipp von Zabern.

Gochal Goral. 1994. A Look at Shik Ngas. Jos: Jos University Press.

Goldenberg Gideon (ed.). 1986. Ethiopian Studies. Proceedings of the Sixth International Conference, Tel-Aviv, 14-17 April 1980. Rotterdam-Boston: A.A. Balkema.

Gordon Cyrus H. 1955. Ugaritic Manual. Roma: Pontificium Institutum Biblicum.

Gordon Cyrus H. 1965. Ugaritic Textbook. Roma: Pontificium Institutum Biblicum.

Gouffé Claude. 1971-1972. "Notes de lexicologie et d'étymologie soudanaises." GLECS 16, 101-119, 155-173.

GragG Gene. 1982. Oromo Dictionary. East Lansing. Michigan: Michigan State University.

Greenberg Joseph H. Studies in African linguistic Classification. Branford, Connecticut: Compass Publishing Company.

Greenberg Joseph H. 1958. "The Labial Consonants of Proto-Afro-Asiatic.” Word 14, 295-302.

Greenberg Joseph H. 1963. "The Languages of Africa.” International Journal of American Linguistics 29.

Griefenow-Mewis Catherine, Voigt Rainer M. (eds.). 1996. Cushitic and Omotic Languages. Proceedings of the Third International Symposium (Berlin, March 17-19, 1994). Köln: Rüdiger Köppe Verlag.

Gromyкo Andrej (ed.). 1988. Proceedings of the Ninth International Congress of Ethiopian Studies (Moscow, 26-29 August 1986). Vol. 5. Moscow: Nauka.

Grottanelli Vinigi L. 1940. Missione etnografica nel Uollega Occidentale. Volume primo. I Mao. Roma: Reale Accademia d'Italia.

Harries Jeanette. 1974. Tamazight Basic Course. Ait Mgild Dialect. Final Report. Madison, Wisconsin: University of Wisconsin.

Haruna Andrew. 1992. Bubbure Lexicon. MS.

Haruna Andrew. 1993. "Hausa and Other Chadic Languages. A Lexical Comparison Between Hausa, Guruntum (Gurnuy), Zaar and Bubbure." Frankfurter Afrikanistische Blätter 5, 75-81.

HAYwARD Richard J. 1984. The Arbore Language: A First Investigation Including a Vocabulary. Hamburg: Helmut Buske Verlag.

Hayward Richard J. 1988. "Remarks on Omotic Sibilants." In: Becchaus-Gerst \& Serzisko 1988: 263-299.

HAYWARD Richard J. 1989. "Comparative Notes on the Language of the S'aamakko." Journal of Afro-Asiatic Languages 1, 1-53.

Hayward Richard J. 1989. “The Notion of 'Default Gender': A Key to Interpreting the Evolution of Certain Verb Paradigms in East Ometo, and Its Implications for Omotic." Afrika und Übersee 72, 17-33.

HaYward Richard J. (ed.). 1990. Omotic Language Studies. London: SOAS.

HAYwARD Richard J. 1997. "Review of Hudson, Grover: Highland East Cushitic Dictionary." Journal of African Languages and Linguistics 18(1), 106-120.

HCVA I-V = Diakonoff Igor, Belova Anna, Militarev Alexander, Porhomovsky Victor, Stolbova Olga. "Historical Comparative Vocabulary of Afrasian.” Part 1-5. St. Petersburg Journal of African Studies 2 (1993), 5-28 \& 3 (1994), 5-26 \& 4 (1994), 7-38 \& 5 (1995), 4-32 \& 6 (1997), 12-35.

HeInE Bernd. 1975. "Notes on the Yaaku Language (Kenya)." Afrika und Übersee 58(2), 119-138.

HeIne Bernd. 1976. "Notes on the Rendille Language." Afrika und Übersee 59, 176-223.

HeInE Bernd. 1977. "Bemerkungen zur Boni-Sprache (Kenia).” Afrika und Übersee 60, 242-295.

HeIne Bernd. 1978. "The Sam Languages. A History of Rendille, Boni and Somali." Afroasiatic Linguistics 6(2), $23-115$.

HeIne Bernd. 1980. The Non-Bantu Languages of Kenya. Berlin: Reimer.

HeIne Bernd. 1982. Boni Dialects. Berlin: Dietrich Reimer Verlag.

Hellwig Birgit. 2000. Goemai - English - Hausa Dictionary. MS. Draft. Printed out on 20 August 2000.

Hetzron Robert (ed.). 1997. The Semitic Languages. London: Routledge.

Hoffmann Carl. 1955. “Zur Sprache der Cibak.” In: LuKas 1955: 118-143.

Hoffmann Carl. 1975. Towards a Comparative Phonology of the Languages of the Angas-Goemai Group. MS. University of Ibadan, faculty seminar on 19 March 1975.

Hoffmann Carl. 1987. "Were There Labial Alveolars and Labial Palatals in Proto-Bura-Margi?” In: JungraithMAYR \& MÜLLER 1987: 451-474. 
Hohenberger Johannes. 1975. "The Nominal and Verbal Afformatives of Nilo-Hamitic and Hamito-Semitic." Abhandlungen für die Kunde des Morgenlandes 42(2).

Hoskison James T. 1983. A Grammar and Dictionary of the Gude Language (Chadic). Ph.D. dissertation. The Ohio State University.

HSED = Orel Vladimir, Stolbova Olga. 1995. Hamito-Semitic Etymological Dictionary. Leiden: E.J. Brill.

Hudson Grover. 1989. Highland East Cushitic Dictionary. Hamburg: Buske.

Hudson Richard. 1996. A Dictionary of Beja. Draft printout of February 29, 1996. Version prepared by R.M. Blench.

Ibriszimow Dymitr. 1990. “Towards a Common Chadic Lexicon.” Zeszyty Naukowe Uniwersytetu Jagiellońskiego. Prace Językoznawcze 102, 1-122.

Ibriszimow Dymitr. 2003. The Verb in Ngamo. MS. Presented at the 2nd Biennal International Colloquium on the Chadic Languages, Prague, 11-12 Oct. 2003.

Ibriszimow Dimitri, Leger Rudolf (eds.). 1995. Studia Chadica et Hamitosemitica. Köln: Rüdiger Köppe Verlag. ILLIČ-SvitYČ Vladislav. 1966. "Iz istorii čadskogo konsonantizma. Labial'nye smyčnye.” In: UsPENSKIJ B.A. (ed.). 1966. Jazyki Afriki. Voprosy struktury, istorii i tipologii. Moskva: Nauka: 9-34.

ILLIČ-SvityČ Vladislav. 1976. Opyt sravnenija nostratičeskih jazykov (semitohamitskij, kartvel'skij, indoevropej-

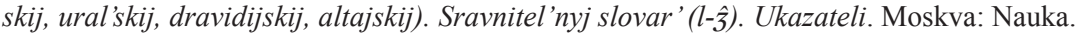

JAGGAR Philip J. 1989. "Guruntum (gùrdùn) (West Chadic-B): Linguistic Notes and Wordlist." African Languages and Cultures 2(2), 175-202.

JEA = Journal of Egyptian Archaeology (Birmingham).

Johnstone T.M. 1977. Harsūsi Lexicon. London: Oxford University Press.

Johnstone T.M. 1981. Jibbāli Lexicon. London: Oxford University Press.

Johnstone T.M. 1987. Mehri Lexicon. London: University of London.

JungRAITHMAYR Herrmann. 1961. "Beobachtungen zur tschadohamitischen Sprache der Jegu (und Jonkor) von Abu Telfan (Republique du Tchad)." Afrika und Übersee 45, 95-123.

JungraithMAYR Herrmann. 1962. Wörterbuch der Angas-Sprache. MS.

JungRAITHMAYR Herrmann. 1962. Wörterverzeichnis der Goemay-Sprache. MS.

JungratthmaYr Herrmann. 1963. "Die Sprache des Sura (Maghavul) in Nordnigerien." Afrika und Übersee 47, $8-89,204-220$.

JungRaithMAYR Herrmann. 1966. Wörterverzeichnis Kanakuru-Deutsch. MS.

JungratthmaYr Herrmann. 1968. "A Comparative Word List of the Ron Languages (Southern Plateau, N. Nigeria).” Africana Marburgensia 1(2), 3-12.

JungraithmaYr Herrmann. 1970. Die Ron-Sprachen. Tschadohamitische Studien in Nordnigerien. Glückstadt: Verlag J.J. Augustin.

JungraithMAYR Herrmann. 1972. Wordlist of Kwang-Mobu. MS. 10-17 Jan. 1972.

JungRAITHMAYR Herrmann. 1973. Dictionnaire birgit-français. MS.

JungratThMAYR Herrmann. 1973. Gadang. Verbes I. MS. March 1973.

JungraithMAYR Herrmann. 1973. Masa (Bongor) Lexicon. MS. Frankfurt-Marburg.

JungraithmaYr Herrmann (ed.). 1982. The Chad Languages in the Hamitosemitic-Nigritic Border Area. Berlin:

Dietrich Reimer.

JungratthMAYR Herrmann. 1990. Lexique mokilko. Berlin: Dietrich Reimer Verlag.

JungRAITHMAYR Herrmann. 1990. Lexique mubi-français (Tchad oriental). MS. Frankfurt a/M.

JUNGRAITHMAYR Herrmann. 1990. Sur les langues sarwa, gadang et miltu (groupe sibine/somray). MS.

JungraithmaYr Herrmann (in collaboration with Njẹnọ Andriya Galadima and Ulrich KLeinewillinghöFer). 1991. A Dictionary of the Tangale Language (Kaltungo, Northern Nigeria) with a Grammatical Introduction. Berlin: Dietrich Reimer Verlag.

JUNGRAITHMAYR Herrmann. 1993. Lexique sibine (sumray) - français. MS. Frankfurt.

JungRAITHMAYR Herrmann. 2004. "Das Birgit, eine osttschadische Sprache - Vokabular und grammatische Notizen.” In: TAKÁCS 2004b: 342-371.

JungRaithmaYr Herrmann, AdAms Abakar. 1992. Lexique migama. Berlin: Dietrich Reimer Verlag.

Jungraithmayr Herrmann, CAprile Jean-Pierre (eds.). 1978. Cinq textes tchadiques (Cameroun et Tchad). Présentation linguistique. Berlin: Dietrich Reimer Verlag.

Jungraithmayr Herrmann, Leger Rudolf. 1993. "The Benue-Gongola-Chad Basin - Zone of Ethnic and Linguistic Compression.” Berichte des Sonderforschungbereichs 268(2), 161-172. 
Jungratthmayr Herrmann, Ibriszimow Dymitr. 1994. Chadic Lexical Roots. Vol. II. Documentation. Berlin: Dietrich Reimer Verlag.

JungraithmaYr Herrmann, Miehe Gudrun (ed.). 1981. Berliner Afrikanistische Vorträge XXI. Deutscher Orientalistentag, Berlin 24.-29. März 1980. Berlin: Verlag von Dietrich Reimer.

Jungraithmayr Herrmann, Müller Walter W. (eds.). 1987. Proceedings of the Fourth International HamitoSemitic Congress. Amsterdam: John Benjamins.

Jungraithmayr Herrmann, Shimizu Kiyoshi. 1981. Chadic Lexical Roots. Vol. II. Tentative Reconstruction, Grading and Distribution. Berlin: Verlag von Dietrich Reimer.

JungratthmaYr Herrmann, Tourneux Henry (eds.). 1990. Études tchadiques. Verbes monoradicaux suivis d'une note sur la negation en haoussa. Actes de la XIIème réunion de Groupe d'Études Tchadiques LACITOCNRS-PARIS. Paris: Librairie Orientaliste Paul Geuthner.

Justinard (?). 1914. Manuel de berbère marocain (dialecte chleuh). Paris: Librairie Orientaliste Paul Geuthner.

Kaye Alan S. (ed.). 1991. Semitic Studies in Honor of Wolf Leslau on the Occasion of His Eighty-Fifth Birthday, November 14th, 1991. Vol. II. Wiesbaden: Otto Harrassowitz.

KB = Koehler Ludwig, Baumgartner Walter. 1994. The Hebrew and Aramaic Lexicon of the Old Testament. I-V. Leiden: E.J. Brill.

KHW $=$ WeSTENDORF Wolfhart. 1977. Koptisches Handwörterbuch. Heidelberg: Carl Winter Universitätsverlag. Kidda Mairo E. 1991. Dera Basic and Cultural Vocabularies. MS. October 1991.

KleinewillinghÖFer Ulrich. 1990. "Monoradical Verbs in Waja.” In: Jungraithmayr \& Tourneux 1990: 229-241.

Kossmann Maarten. 1999. Essai sur la phonologie du proto-berbère. Köln: Rüdiger Köppe Verlag.

Kraft Charles H. 1981. Chadic Wordlists. I-III. Berlin: Dietrich Reimer Verlag.

Labahn Thomas (ed.). 1984. Proceedings of the Second International Congress of Somali Studies. Vol. 1. Hamburg: Buske Verlag.

LAMBerti Marcello. 1986. Die Somali-Dialekte. Eine Vergleichende Untersuchung. Hamburg: Helmut Buske Verlag.

LAMBERTI Marcello. 1993. Die Shinassha-Sprache. Materialien zum Boro. Heidelberg: Carl Winter Universitätsverlag.

LAmberTi Marcello. 1993. Materialien zum Yemsa. Heidelberg: Carl Winter Universitätsverlag.

LAmberti Marcello, Sottile Roberto. 1997. The Wolaytta Language. Köln: Rüdiger Köppe Verlag.

Lamberti Marcello, Tonelli, Livia (eds.). 1999. Afroasiatica Tergestina. Papers from the 9th Italian Meeting of Afro-Asiatic (Hamito-Semitic) Linguistics, Trieste, April 23-24, 1998. Contributi presentati al $9^{\circ}$ Incontro di Linguistica Afroasiatica (Camito-Semitica), Trieste, 23-24 Aprile 1998. Padova: Unipress.

Lanfry le P. Jacques. 1973. Ghadames. II. Glossarie. Alger: Le Fichier Périodique.

Laoust Émile. 1918. Étude sur le dialecte berbère des Ntifa. Grammaire, textes. Paris: Ernest Leroux.

Laoust Émile. 1931. Siwa. I. Son parlier. Paris: Librairie Ernest Leroux.

Laoust Émile. 1942. Contribution à une étude de la toponymie du Haut Atlas. Paris: Paul Geuthner.

LeBeuf Jean-Paul. 1942. "Vocabulaires kotoko: Makari, Goulfeil, Kousseri, Afadé." Bulletin de l'Institut Français d'Afrique Noire 4, 160-174.

LegER Rudolf. 1992. "Sprachproblem aus dem Westtschadischen. Kupto- und Kwamitexte." Afrikanistische Arbeitspapiere 28, 5-32.

LEgER Rudolf. 1993. "Die Geschichte der Kwami nach einer Erzählung von Yerma Buba mit grammatischen Erläuterungen." Mitteilungen des Sonderforschungs-bereichs 268, 143-177.

Lenssen Tilman. 1982. Das Verb im Kwang (Tschad) - eine phonologische Studie. M.A. thesis. Marburg: PhilippsUniversität Marburg.

Lenssen Tilman. 1984. "Studien zum Verb im Kwang (Tschad)." Africana Marburgensia Sonderheft 8.

LESLAU Wolf. 1938. Lexique soqotri (sudarabique moderne), avec comparaisons et explications étymologiques. Paris: Librairie C. Klincksieck.

LeSLAu Wolf. 1945. Gafat Documents. Records of a South-Ethiopic Language. Grammar, Text and Comparative Vocabulary. New Haven, Connecticut: American Oriental Society.

LESLAu Wolf. 1956. Étude descriptive et comparative du gafat (éthiopien méridional). Paris: Librairie C. Klincksieck.

LesLAu Wolf. 1959. A Dictionary of Moča (Southwestern Ethiopia). Berkeley-Los Angeles: University of California Press.

LESLAU Wolf. 1963. Etymological Dictionary of Harari. Berkeley-Los Angeles: University of California. 
Leslau Wolf. 1969. "Southeast Semitic Cognates to the Akkadian Vocabulary." III. Journal of the American Oriental Society 89, 18-22.

Leslau Wolf. 1979. Etymological Dictionary of Gurage (Ethiopic). Vol. III. Etymological Section. Wiesbaden: Otto Harrassowitz.

LesLau Wolf. 1980. "Proto-Sidamo *z." Afrika und Übersee 53, 119-129.

Leslau Wolf. 1982. "North Ethiopic and Amharic Cognates in Tigre." Annali, Istituto Universitario Orientale di Napoli, supplemento 31, vol. 42, fasc. 2, 1-85.

Leslau Wolf. 1986. “Cushitic Loanwords in Gurage.” In: Goldenberg 1986: 373-387.

LesLau Wolf. 1987. Comparative Dictionary of Ge ez (Classical Ethiopic). Wiesbaden: Otto Harrassowitz.

LesLau Wolf. 1988. "Observations on Sasse's Vocabulary of Burji." Afrika und Übersee 71, 177-203.

Levy Jacob. 1924. Wörterbuch über die Talmudim und Midraschim nebst Beiträgen von Heinrich Leberecht Fleischer. Zweite Auflage mit Nachträgen und Berichtigungen von Lazarus Goldschmidt. I-IV. BerlinWien: Benjamin Harz Verlag.

Loubignac Victorien. 1924. Étude sur le dialecte berbère des Zaïan et Aït Sgougou. Textes (deuxième section). Paris: Ernest Leroux.

LöHr Doris. 2002. Die Sprache der Malgwa (Nárá Málgwa). Grammatische Erstbeschreibung einer zentralsudanischen Sprache Nordost-Nigerias. Frankfurt am Main: Peter Lang Europäischer Verlag der Wissenschaften.

Löwinger S., Somogyi J. (eds.). 1948. Ignace Goldziher Memorial Volume. Part I. Budapest: Globus.

LuKas Johannes. 1936. "Die Logone-Sprache im Zentralen Sudan." Abhandlungen für die Kunde des Morgenlandes 21(6).

LuKas Johannes. 1937. "Zentralsudanische Studien.” Abhandlungen aus dem Gebiet der Auslandskunde. Hansische Universität. Reihe B, Band 45, Band 24.

LuKAs Johannes. 1939. "Die Sprache des Buduma in Zentralen Sudan." Abhandlungen für die Kunde des Morgenlandes 24(2).

LuKAS Johannes. 1941. Deutsche Quellen zur Sprache der Musgu in Kamerun. Berlin: Dietrich Reimer Verlag.

LuKAS Johannes (ed.). 1955. Afrikanistische Studien. Berlin: Akademie-Verlag.

LuKAs Johannes. 1966. "Tschadohamitische Sprachproben aus Nordnigerien (Karekare- und Bolanci-Texte)." In: LuKAS 1966 (ed.): 173-207.

LuKas Johannes (ed.). 1966. Neue afrikanistische Studien. Hamburg: Deutsches Institut für Afrika-Forschung.

LuKAs Johannes. 1970. Studien zur Sprache der Gisiga (Nordkamerun). Hamburg: Verlag J.J. Augustin.

LuKAs Johannes. 1971. "Die Personalia und das primäre Verb im Bolanci (Nord-nigerien). Mit Beiträge über das Karekare." Afrika und Übersee 55, 114-139.

Lukas Johannes. 1974-1975. "Studien zur Bade-Sprache (Nigeria)." Afrika und Übersee 58(2), 82-105.

LuKAs Johannes. 1977. "Tschadische Studien I. Beiträge zur Kenntnis des Mokulu.” Afrika und Übersee 60, 1-58, $182-229$.

Maghway J.B. 1989. "Iraqw Vocabulary." Afrikanistische Arbeitspapiere 18, 91-118.

Magwa, rev. JoshuA G. et al. (20 members of the "Ron Language Committee"). 1985. A Ron Alphabet. Jos, Nigeria: Nigeria Bible Translation Trust.

Marcus Harold (ed.). 1994. New Trends in Ethiopian Studies. Vol. 1. Lawrenceville: Red Sea Press.

Matsushita Shuji. 1972. An Outline of Gwandara Phonemics and Gwandara-English Vocabulary. Tokyo: Tokyo Press.

MeInHof Carl. 1906. "Linguistische Studien in Ostafrika. Fortsetzung." Mitteilungen des Seminars für Orientalische Sprachen 9, 278-333.

MerCier Henri. 1937. Vocabulaires et textes berbères dans le dialecte des Ait Izdeg. Rabat: René Céré.

MilitaRev Aleksandr. 1991. "Istoričeskaja fonetika i leksika livijsko-guančskih jazykov.” In: Solncev 1991: 238-267.

Militarev Aleksander. 2005. "Once More About Glottochronology and the Comparative Method: the OmoticAfrasian Case." Orientalia et Classica, Papers of the Institute of Oriental and Classical Studies, Russian State University for the Humanities 6, 339-408.

MiRT Heide. 1970-1971. "Zur Morphologie des Verbalkomplexes im Mandara." Afrika und Übersee 54(1-2), $1-76$.

Moreno Martino Mario. 1938. Introduzione alla lingua ometo. Milano: Mondadori.

Moreno Martino Mario. 1940. Manuale di sidamo. Grammatica, esercizi, testi, glossario. Roma: Casa Editrice A. Mondadori. 
Mouchet Jean. 1950. "Vocabulaires comparatifs de quinze parlers du Nord-Cameroun.” Bulletin de la Société d'Études Camerounaises 29-30, 5-74.

Mouchet Jean. 1953. "Vocabulaires comparatifs de sept parlers du Nord-Cameroun." Bulletin de la Société d'Études Camerounaises 41-42, 136-206.

Mouchet Jean. 1966. Le parler daba: esquisse grammaticale précédée d'une note sur l'ethnie daba, suivie de lexiques daba-français et français-daba. Yaoundé: R.E.C.

Mous Maarten. 1996. "Was There Ever a Southern Cushitic Language (Pre-)Ma'a?" In: Griefenow-Mewis \& VoIGT 1996: 201-211.

Mous Maarten, QORRo Martha, Kiessuing Roland. 2002. Iraqw-English Dictionary with an English and a Thesaurus Index. Köln: Rüdiger Köppe Verlag.

MuKARovsKy Hans G. 1969. "Baskisch-berberische Entsprechungen.” Wiener Zeitschrift für die Kunde des Morgenlandes $62,32-51$.

Mukarovsky Hans G. 1981. "Einige hamitosemitische und baskische Wortstämme.” In: JungraithmaYr \& Miehe 1981: 105-118.

Mukarovsky Hans G. 1981.” Wo steht das Saharische?” Afrika und Übersee 64, 187-226.

Mukarovsky Hans G. 1982. "Lateinische Lehnwörter im Hausa.” In: JungraithmaYr 1982: 261-268.

Mukarovsky Hans G. 1987. Mande-Chadic Common Stock. A Study of Phonological and Lexical Evidence. Wien: Afro-Pub.

Mukarovsky Hans G. 1989. On the Relations of Cushitic, Omotic and Chadic Languages. MS. Handout for the 2nd International Symposium on Cushitic and Omotic Languages, Torino, 1989. Proceedings forthcoming.

MÜLLER Friedrich. 1886. "Die Musuk-Sprache in Central-Afrika. Nach den Aufzeich-nungen von Gottlob Adolf Krause herausgegeben." Sitzungsberichte der Kaiser-lichen Akademie der Wissenschaften. Phil.-hist. Klasse 112(1), 353-421.

MÜlLER Walter W. 1963. “Altsüdarabische Beiträge zum hebräischen Lexikon.” Zeitschrift für die Alttestamentliche Wissenschaft 75(3), 304-316.

MülLER Walter W. 1981. Review of LesLau W. "Etymological Dictionary of Gurage (Ethiopia)." Zeitschrift der Deutschen Morgenländische Gesellschaft 131(2), 396-404.

NaKano Aki'o. 1986. Comparative Vocabulary of Southern Arabic - Mahri, Gibbali and Soqotri. Tokyo: Institute for the Study of Languages and Cultures of Asia and Africa.

NAİT-ZeRRAD Kamal. 1998. Dictionnaire des racines berbères (formes attestées). I: $a$-b $b$ l. Paris-Louvain: Peeters.

NeHLIL. 1909. Étude sur le dialecte de Ghat. Paris: Éditions Ernest Leroux.

NetTing Robert M. 1967. Kofyar Vocabulary. MS.

Newman Paul. 1964. "A Word List of Tera.” Journal of West African Languages 1(2), 33-50.

Newman Paul. 1970. "Historical Sound Laws in Hausa and Dera (Kanakuru)." Journal of West African Languages $7(1), 39-51$.

Newman Paul. 1974. The Kanakuru Language. Leeds: The Institute of Modern English Language Studies, University of Leeds in association with The West African Linguistic Society.

Newman Paul. 1977. "The Formation of the Imperfective Verb Stem in Chadic.” Afrika und Übersee 60(3), 178192.

Nicolas Francis. 1953. La langue berbère de Mauritanie. Dakar: Institut Français d'Afrique Noire.

Nicolas Francis. 1957. "Vocabulaires ethnographiques de la Tamâjeq des Iullemmeden de l'est (Touâreg de la Colonie du Niger, Afrique Occidentale Française)." Anthropos 52, 49-63, 564-580.

Orel Vladimir. 1995. "Semitohamitskij, sinokavkazskij, nostratičeskij." Moskovskij Lingvističeskij Žurnal 1, 99-116.

Ormsby G. "Notes on the Angass Language." Journal of the Royal African Society 12 (1912-1913), 421-424 \& 13 (1913-1914), 54-61, 204-210, 313-315.

PARADISI Umberto. 1960. "Il berbero di Augila. Materiale lessicale." Rivista degli Studi Orientali 35(3-4), 157177.

Parker Enid M., Hayward Richard J. 1985. An Afar-English-French Dictionary (with Grammatical Notes in English). London: School of Oriental and African Studies, University of London.

Pearce Mary. 1998-1999. "Consonant and Tone in Kera (Chadic)." Journal of West African Languages 27(1), 33-70.

Pellat Charles. 1955. Textes berbères dans le parler des Aït Seghrouchen de la Moulouya. Paris: Éditions Larose. Penchoen Thomas G. 1973. Tamazight of the Ait Ndhir (Afroasiatic Dialects, Vol. 1). Los Angeles: Undena Publications. 
Pillinger Steve, Galboran Letiwa. 1999. A Rendille Dictionary. Köln: Rüdiger Köppe Verlag.

Pilszczikowa Nina. 1958. "Contribution à l'étude des rapports entre le haoussa et les autres langues du groupe nigéro-tchadien." Rocznik Orientalistyczny 22(2), 75-99.

PorhomovskiJ Viktor (ed.). 1987. Afrikanskoe istoričeskoe jazykoznanie. Problemy rekonstrukcii. Moskva: Nauka.

Prasse Karl-G. 1969. "A propos de l'origine de h touareg (tahaggart)." Det Kongelige Danske Videnskabernes Selskab. Historisk-filosofiske Meddelelser 43(3).

Prasse Karl-G. 1972. Manuel de grammaire touaregue. I-III. Phonétique - écriture - pronom. Copenhague: Université de Copenhague.

Prasse Karl-G., Alojaly Ghoubeïd, Mohamed Ghabdouane. 1998. Lexique touareg-français. Copenhague: Museum Tusculanum Press, Université de Copenhague.

Prasse Karl-G., Alojaly Ghoubeïd, Mohamed Ghabdouane. 2003. Dictionnaire touareg-français (Niger). Copenhagen: Museum Tusculanum Press, University of Copenhagen.

Pweddon Nicholas Nzovudeto (edited by SKInNER Neil). 2000. Bwatiye-English Dictionary with English-Bwatiye Index. Madison, Wisconsin: Dr. Nicholas Pweddon.

RABIN Chaim. 1970. "La correspondance d hébreu - $\underline{d}$ arabe." In: CoHEN 1970: 290-297.

Rapp Eugen L., Benzing Brigitta. 1968. Dictionary of the Glavdá Language. Frankfurt am Main: Bible Society Franfurt am Main.

$\mathrm{RdE}=$ Revue d'Égyptologie (Paris).

ReINISCH Leo. 1884. "Die Chamirsprache in Abessinien. II. Chamir-deutsches Wörterbuch." Sitzungsberichte der Kaiserlichen Akademie der Wissenschaften. Phil.-hist. Classe 106, 330-450.

ReINISCH Leo. 1885. "Die Quarasprache in Abessinien. II. Quarisch-deutsches Wörterbuch." Sitzungsberichte der Kaiserlichen Akademie der Wissenschaften. Phil.-hist. Classe 109(1), 3-152.

ReInIsch Leo. 1886. "Die `Afar-Sprache. II." Sitzungsberichte der Kaiserlichen Akademie der Wissenschaften. Phil.-hist. Classe 113(2), 795-916.

ReInIsch Leo. 1887. "Die `Afar-Sprache. III. Deutsch-`Afarsches Wörter-verzeichnis.” Sitzungsberichte der Kaiserlichen Akademie der Wissenschaften. Phil.-hist. Classe 114(1), 89-168.

ReInIsch Leo. 1887. Wörterbuch der Bilin-Sprache. Wien: Alfred Hölder Verlag.

ReINISCH Leo. 1888. "Die Kafa-Sprache in Nordost-Afrika. II. Kafa-Deutsches Wörterbuch.” Sitzungsberichte der Kaiserlichen Akademie der Wissenschaften. Phil.-hist. Classe 116, 251-386.

ReInIsch Leo. 1890. Wörterbuch der Saho-Sprache. Wien: Alfred ReInISCh Leo. 1887. Wörterbuch der BilinSprache. Wien: Alfred Hölder Verlag.

ReINISCH Leo. 1895. Wörterbuch der Bedawye-Sprache. Wien: Alfred Hölder Verlag.

ReInISCh Leo. 1902. Die Somali-Sprache. II. Wörterbuch. Wien: Alfred Hölder Verlag.

REINISCH Leo. 1904. "Der Dschäbärtidialekt der Somalisprache." Sitzungsberichte der Kaiserlichen Akademie der Wissenschaften 148(5), 1-88.

Renisio Amédée. 1932. Étude sur les dialectes berbères des Beni Iznassen, du Rif et des Senhaja de Sraïr. Grammaire, textes et lexique. Paris: Éditions Ernest Leroux.

Reutt T.E., Kogan E. Z. 1973. "Materialy po leksike jazykov margi i bura.” In: Bespis'mennye i mladopis'mennye jazyki Afriki. Moskva: Nauka, 83-147.

Roper E.-M.. 1928. Tu Bedawic. An Elementary Handbook for the Use of Sudan Government Officials. Hertford: Stephen Austin \& Sons.

Rossing Melvin Olaf. 1978. Mafa-Mada: A Comparative Study of Chadic Languages in North Cameroun. Ph.D. dissertation. Wisconsin: University of Wisconsin-Madison.

Sabar Yona. 2002. A Jewish Neo-Aramaic Dictionary. Wiesbaden: Harrassowitz.

SACHnine Michka. 1976. "Notes sur le zime (lame) parlé au Cameroun." Africana Marburgensia 9(1), 71-86.

SACHnine Michka. 1982. Dictionnaire lamé-français. Lexique français-lamé. Paris: SELAF.

SASSE Hans-Jürgen. 1973. "Spuren von Pharyngalen im Galab." Afrika und Übersee 56, 266-275.

SASSE Hans-Jürgen. 1974. "Notes on the Structure of Galab." Bulletin of the School of Oriental and African Studies $37,407-438$.

SASSE Hans-Jürgen. 1975. "Galla/š/, /s/ und /f/." Afrika und Übersee 58, 244-263.

SASSE Hans-Jürgen. 1979. "The Consonant Phonemes of Proto-East-Cushitic (PEC).” Afroasiatic Linguistics 7(1), $1-67$.

SAsse Hans-Jürgen. 1981. “Neue Perspektiven im Afroasiatischen?” In: JungraithmaYr \& Miene 1981: 145-165.

SAsSE Hans-Jürgen. 1982. An Etymological Dictionary of Burji. Hamburg: Helmut Buske Verlag.

SAVÀ Graziano. 2005. English-Ts'amakko Wordlist. MS. 
SAXon Douglas Esche. 1982. "Linguistic Evidence for the Eastward Spread of Ubangian Peoples." In: EhrEt \& POSNANSKY 1982: 66-77.

SchleE Günther. 1978. Sprachliche Studien zum Rendille: Grammatik, Texte, Glossar. Hamburg: Helmut Buske Verlag.

Schuн Russell G. 1978. "Bade/Ngizim Vowels and Syllable Structure." Studies in African Linguistics 9(3), $247-$ 283.

Schuн Russell G. 1981. A Dictionary of Ngizim. Berkeley, California: University of California.

Schuн Russell G. 1984. "West Chadic Vowel Correspondences.” In: Bynon 1984: 167-223.

$\mathrm{SD}=$ Beeston A.F.L., Ghul Mahmud `Ali, Müller Walter W., Ryckmans Gonzague. 1982. Sabaic Dictionary (English-French-Arabic). Dictionnaire sabéen (anglais-français-arabe). Louvain-la-Neuve-Beyrouth: Peeters, Librairie du Liban.

SED I = Militarev Alexander, Kogan Leonid. 2000. Semitic Etymological Dictionary. Vol. 1. Anatomy of Man and Animals. Münster: Ugarit-Verlag.

Segert S., Bodrogligeti András J.E. (eds.). 1983. Ethiopian Studies Dedicated to Wolf Leslau. Wiesbaden: Otto Harrassowitz.

SeIBERT Uwe. 2000. Comparative Ron Wordlist. MS. Frankfurt.

Setнe Kurt. 1959. Ägyptische Lesestücke zum Gebrauch im akademischen Unterricht. Texte des Mittleren Reiches. ${ }^{3}$ Hildesheim: Georg Olms.

Shimizu Kiyoshi. 1975. Boghom and Zaar: Vocabulary and Notes. MS. Kano.

Shimizu Kiyoshi. 1978. "The Southern Bauchi Group of Chadic Languages. A Survey Report." Africana Marburgensia. Special Issue 2, 1-50.

SiEBerT Ralph. 1994. "Languages of the Abbaya/Chamo Area - Report Part I (with Notes on Koorete by L. Hoeft)." Survey of Little-Known Languages of Ethiopia (S.L.L.E.) Reports 21, 1-24.

Siebert Ralph. 1994-1995. "A Survey of the Male Language.” Survey of Little-Known Languages of Ethiopia (S.L.L.E.) Reports 24, 1-12.

Siebert Ralph, Wedekind Claus. 1994. "Third S.L.L.E. Survey on Languages of the Begi/Asosa Area." Survey of Little-Known Languages of Ethiopia (S.L.L.E.) Reports 15, 1-19.

Simeone-Senelle Marie-Claude. 1997. "The Modern South Arabian Languages.” In: Hetzron 1997: 378-423.

Simeone-Senelle Marie-Claude, Lonnet Antoine. 1991. "Lexique soqotri: les noms des parties du corps." In: KAYE 1991: 1443-1487.

SirLINGER Eugene. 1937. Dictionary of the Goemay Language. Jos, Nigeria: Prefecture Apostolic of Jos.

SiSAJa I = D'Jakonov Igor', Belova Anna, Četveruhin Aleksandr, Militarev Aleksandr, Porhomovskij Viktor, Stolbova Ol'ga. 1981. "Sravnitel'no-istoričeskij slovar' afrazijskih jazykov. Vypusk 1. p-p-b-f." In: Pis'mennye pamjatniki i problemy istorii kul 'tury narodov Vostoka. XV godičnaja naučnaja sessija Leningradskogo Otdelenija Instituta Vostokovedenija Akademii Nauk SSSR. Moskva: Nauka, 3-127.

SiSAJa II = D'Jakonov Igor', Belova Anna, Militarev Aleksandr, PorhomovskiJ Viktor, Stolbova Ol'ga. 1982. "Sravnitel'no-istoričeskij slovar' afrazijskih jazykov. Vypusk 2. t-ț-d.". In: Pis'mennye pamjatniki i problemy istorii kul tury narodov Vostoka. XVI godičnaja naučnaja sessija Leningradskogo Otdelenija Instituta Vostokovedenija Akademii Nauk SSSR. Moskva: Nauka, 3-93.

SiSAJa III = D'Jakonov Igor', Belova Anna, Militarev Aleksandr, Porhomovskij Viktor, Stolbova Ol'ga. 1986. „Sravnitel'no-istoričeskij slovar' afrazijskih jazykov. Vypusk 3. s-c-c̣-3, č-č-ž, ŝ-ĉ-ĉ̣ s labialami.” In: Pis 'mennye pamjatniki i problemy istorii kul tury narodov Vostoka. XVI godičnaja naučnaja sessija Leningradskogo Otdelenija Instituta Vostokovedenija Akademii Nauk SSSR. Moskva: Nauka, 3-46.

SkinNer Margaret G. 1979. Aspects of Pa'anci Grammar. Ph.D. thesis. Madison: University of Wisconsin, Madison.

SkinNER Neil. 1977. "North Bauchi Chadic Languages: Common Roots.” Afroasiatic Linguistics 4(1), 1-49.

SKInNER Neil. 1996. Hausa Comparative Dictionary. Köln: Rüdiger Köppe Verlag.

SöLKen Heinz. 1967. Seetzens Áffadéh. Ein Beitrag zur Kotoko-Sprachdokumentation. Berlin: Akademie-Verlag.

Solncev V.M. (ed.). 1991. Jazyki Azii i Afriki. IV, kniga 2. Moskva: Glavnaja Redakcija Vostočnoj Literatury.

SotTile Roberto. 1999. “The Consonant System of Gamu.” In: Lamberti \& Tonelli 1999: 427-446.

Stolbova Olga. 1996. Studies in Chadic Comparative Phonology. Moscow: "Diaphragma" Publishers.

Stolbova Olga. 1977. "Opyt rekonstrukcii verhnezapadnočadskih kornej.” In: Jazyki zarubežnogo Vostoka. Sbornik statej. Moskva: Nauka, 152-160.

Stolbova Ol'ga. 1987. "Sravnitel'no-istoričeskaja fonetika i slovar' zapadno-čadskih jazykov.” In: PorHomovsKIJ 1987: 30-268. 
Stolbova Olga. 1994. Akkadian-Chadic Cognates. MS. Paper presented at the 6th International Hamito-Semitic Congress, Moscow, April 1994. Proceedings forthcoming.

Stolbova Olga. 1996. Studies in Chadic Comparative Phonology. Moscow: "Diaphragma" Publishers.

Stroomer Harry. 1987. A Comparative Study of Three Southern Oromo Dialects in Kenya. Hamburg: Buske.

Stroomer Harry. 1995. A Grammar of Borana Oromo (Kenya). Phonology, Morphology, Vocabularies. Köln: Köppe.

Stroomer Harry. 2000. “An Early European Source on Berber: Chamberlayne (1715).” In: Chaker \& Zaborski 2000: 303-316.

StRÜMPELl Kurt. 1910. "Vergleichendes Wörterverzeichnis der Heidensprachen Adamauas.” Zeitschrift für Ethnologie 42, 444-488.

STRÜMPELl Kurt. 1922-1923. "Wörterverzeichnis der Heidensprachen des Mandara-Gebirges (Adamaua)." Zeitschrift für Eingeborenen-Sprachen 13, 109-149.

SudLow David. 2001. The Tamasheq of North-East Nurkina Faso. Notes on Grammar and Syntax Including a Key Vocabulary. Köln: Köppe.

TAïFI Miloud. 1991. Dictionnaire tamazight-français (parlers du Maroc central). Paris: L'Harmattan-Awal.

TAKÁCS Gábor. 1999. "Sibilant and Velar Consonants of South Cushitic and Their Regular Correspondences in Egyptian and Other Afro-Asiatic Branches." In: LAMBERTI \& TONELli 1999: 393-426.

TAKÁCS Gábor. 2004. Comparative Dictionary of the Angas-Sura Languages. Berlin: Dietrich Reimer Verlag.

TAKÁcs Gábor (ed.). 2004. Egyptian and Hamito-Semitic Studies in Memoriam W. Vycichl. Leiden: Brill.

TAL Abraham. 2000. A Dictionary of Samaritan Aramaic. Leiden: Brill.

Tosco Mauro. 1991. A Grammatical Sketch of Dahalo. Hamburg: Helmut Buske Verlag.

Tosco Mauro. 1994. The Historical Reconstruction of a Southern Somali Dialect: Proto-Karre-Boni. MS. Kindly submitted by the author in 1996. Published in: Sprache und Geschichte in Afrika 15, 153-209.

Tosco Mauro. 2001. The Dhaasanach Language. Grammar, Texts, Vocabulary of a Cushitic Language of Ethiopia. Köln: Köppe.

Toselu Fr. Giovanni. 1939. Elementi di lingua Magi. Grammatica e dizionario con alcuni cenni sulla popolazione Magi. Torino: Istituto Missioni Consolata.

Tourneux Henry. 1977. "Une langue tchadique disparue: le muskum.” Africana Marburgensia 10(2), 13-34.

Tourneux Henry. 1978. Le mulwi ou vulum de Mogroum (Tchad). Langue du groupe musgu - famille tchadique. Paris: Centre National de la Recherche Scientifique.

Tourneux Henry. 1978. "Racine verbale en mulwi.” In: CAPrile \& JungratthmaYr 1978: 89-93.

Tourneux Henry, Seignobos Christian, Lafarge Francine. 1986. Les Mbara et leur langue (Tchad). Paris: Société d'Études Linguistiques et Anthropologiques de France.

Tourneux Henry. 1987. "Note complementaire sur les baldamu et leur langue." Africana Marburgensia 20(1), $52-58$.

Tourneux Henry. 1991. Lexique pratique du munjuk des rizières. Dialecte de Pouss. Paris: Librairie Orientaliste Paul Geuthner.

Tucker Archibald N., Bryan Margaret A. 1974. "The 'Mbugu' Anomaly.” Bulletin of the School of Oriental and African Studies 37, 188-207.

UspensKiJ B.A. (ed.). 1966. Jazyki Afriki. Voprosy struktury, istorii i tipologii. Moskva: Nauka.

Vergari Moreno, Vergari Roberta. 2003. A Basic Saho-English-Italian Dictionary. Asmara, Eritrea (publisher not indicated).

Voigt Rainer M. 1998. "Vergleichende Tschadistik heute - Fortschritte in der tschadischen und semitohamitischen Komparatistik. Besprechung von Herrmann JungraithmaYR \& Dymitr Ibriszimow: Chadic Lexical Roots. Vol. I-II." Orientalistische Literaturzeitung 93(6), 607-619.

Vycichl Werner (ed. by Dymitr Ibriszimow \& Kossmann Maarten). 2005. Berberstudien \& A Sketch of Siwi Berber (Egypt). Köln: Köppe Verlag.

WARD William A. 1981. "Lexicographical Miscellanies II." Studien zur Altägypt-ischen Kultur 9, 359-373.

$\mathrm{Wb}=$ ERMAN Adolf, GraPOW Hermann. 1957-1971. Wörterbuch der ägyptischen Sprache. I-V. ${ }^{2}$ Berlin: Akademie-Verlag.

Wedekind Klaus. 1990. "Gimo-Jan or Ben-Yem-Om: Benč-Yemsa Phonemes, Tones, and Words.” In: HaYward 1990: 68-141.

WeHR Hans. 1952. Arabisches Wörterbuch für die Schriftsprache der Gegenwart. Leipzig: Otto Harrassowitz. Weibegué Christophe, Palayer Pierre. 1982. Lexique lele-français. Sarh, Tchad: Centre d'Études Linguistiques. Whiteley Wilfred H. 1953. Studies in Iraqw. Kampala: East African Institute of Social Research. 
Whiteley Wilfred H. 1958. A Short Description of Item Categories in Iraqw (with Material on Gorowa, Alagwa and Burunge). Kampala: East African Institute of Social Research

WoLfF Ekkehard. 1971. "Die sprachliche Situation im Gwoza-Distrikt (Nordostnigeria)." Journal of African Languages 10(1), 61-74.

WolfF Ekkehard. 1972. Die Verbalphrase des Laamang (Nordostnigeria). Eine Studie zur Morphologie tschadischer Sprachen. Ph.D. Diss. Universität zu Hamburg.

WoLfF Ekkehard. 1974. "Neue linguistische Forschungen in Nordostnigeria." Afrika und Übersee 58(1), 7-27.

Wolff Ekkehard, Meyer-Bahlburg H. (eds.). 1983. Studies in Chadic and Afro-Asiatic Linguistics. Hamburg: Helmut Buske Verlag.

WUS = AistLeITNER Josef. 1963. "Wörterbuch der ugaritischen Sprache." Berichte über die Verhandlungen der Sächsischen Akademie der Wissenschaften zu Leipzig. Phil.-hist. Klasse 106(3).

$\mathrm{ZA}=$ Zeitschrift für Assyriologie.

ZABORsKI Andrzej. 1971. "Biconsonantal Verbal Roots in Semitic." Zeszyty Naukowe Universytetu Jagiellońskiego, Prace językoznawcze 5, 51-98.

Allatum die 29 mensis Februarii anno 2012 